\title{
La valutazione dei costi: uno studio applicato all'unità operativa di diagnostica per immagini
}

Salvatore Nieddu§, Maurizio Bianco*

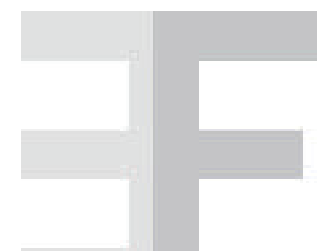

METODI

\begin{abstract}
The aim of the analysis was to evaluate the reasons that caused the poor economic results and performances of a Radiology Department located in a public hospital.

The models of Decision Support System were utilized in order to conduct the studies. These models represent clearly a current economic situation and allow simulations in order to estimate in a preventive way the economic results of different alternative actions.

The economic analysis was conducted according to the extended direct costing method utilizing a single performance (product) as a calculation unit.

The simulations were carried out in accordance to the "price-cost-volume analysis" method that identifies the break even point for the families of products.

These analysis resulted in a significant improvement of the awareness relative to the economic actions of the operative managerial decisions: the main factor in the process of the decision making for the public healthcare structure is to respond to the needs of the potential users and therefore to satisfy the demand, the increase of which is immediately signalised by the lengthening of the awaiting list.

For the structure which is incorporated in the hospital frame there is the necessity to provide the service towards inside the hospital both relative to the patients and to the performances of first aid supported by the emergency department.

It is important to stress that, even if limited or conditional, there is a space for improvement: the first step in this direction is to analyse in order to recognize and to measure, if not able to improve that what is unknown and not measured before.
\end{abstract}

Farmeconomia e percorsi terapeutici 2001; 2 (2): 69-90

\section{INTRODUZIONE}

L'obiettivo dello studio è stato l'approfondimento delle cause che determinavano un risultato economico non soddisfacente relativamente ad un'unità operativa di Diagnostica per Immagini inserita in un presidio ospedaliero di un'azienda territoriale ed è stato perseguito conducendo un'analisi economica dettagliata della stessa.

In particolare occorreva appurare se il disequilibrio del reparto fosse da considerarsi strutturale - a causa della bassa remuneratività delle tariffe del nomenclatore in uso che potevano per determinate prestazioni seppur in condizioni di efficienza, non consentire un' adeguata copertura dei costi del servizio - o se erano presenti aspetti migliorabili, quali:

- mix di produzione, le variazioni dei volumi di produzione degli esami erano infatti prese senza una reale conoscenza dell'impatto economico di tali decisioni;

- presenza di "colli di bottiglia" tecnologici o organizzativi tali da impedire l'ottimizzazione delle attività;

insaturazione o, al contrario, sovraccarico delle risorse tecnologiche o umane;

eccessiva attenzione, nelle decisioni di gestione, ad alcune variabili a scapito di altre: qualità, volumi di prestazioni, tempi di attesa, ricavi e costi.

\section{MATERIALI E METODI}

Nella conduzione dello studio sono stati utilizzati modelli a supporto dei processi decisionali che rappresentano in modo chiaro la situazione economica attuale e che consentono, mediante opportune simulazioni, di valutare in via preventiva i risultati economici di diverse alternative d'azione.

L'analisi economica è stata effettuata utilizzando la configurazione del costo diretto e adottando come oggetto di calcolo la singola prestazione (o prodotto): 1'andare oltre la determi-
§ Università di Torino; Nagima Divisione Sanità, Torino

* Nagima Divisione Sanità, Torino 
nazione di generiche relazioni applicate a livello di unità operativa spingendo l'analisi fino alla determinazione di un margine analitico a livello di singolo prodotto ha permesso di effettuare simulazioni con un elevato grado di analiticità sulle possibili conseguenze di variazioni del mix di produzione.

Per effettuare tali simulazioni è stata utilizzata la metodologia dell' "analisi prezzi/costi/ volumi", ricercando le relazioni tra le seguenti grandezze:

- ricavi, prendendo in considerazione tariffe, quantità prodotte e mix di produzione;

- costi variabili, variabilizzando in questo caso i costi diretti di prodotto;

- costi fissi, considerando come tali i costi indiretti di prodotto e principalmente gli ammortamenti dei macchinari utilizzati per ogni famiglia di prodotti, allo scopo di evidenziare i vincoli o le opportunità legate alla capacità produttiva.

Determinante per il buon esito dello studio è stata la precisione e la qualità dei dati nonché la piena e fattiva collaborazione del responsabile dell'unità operativa di Diagnostica per Immagini, e del responsabile dell'unità operativa di Controllo di Gestione.

\section{RISULTATI}

Il risultato più significativo dello studio è stato il miglioramento della "consapevolezza" dei risvolti economici delle scelte di gestione operativa: nella presa di tali decisioni infatti il fattore prioritario è costituito per una struttura sanitaria pubblica dalla necessità di dare risposta ai bisogni dell'utenza e di soddisfare quindi una domanda il cui incremento è immediatamen- te segnalato dall'allungamento delle liste di attesa. Per una struttura inserita in un presidio ospedaliero esiste inoltre la irrinunciabile necessità di fornire un servizio verso l'interno dell'ospedale sia per quanto riguarda i pazienti ricoverati sia per quanto riguarda le prestazioni d'urgenza in appoggio al Pronto Soccorso.

In particolare le prestazioni per interni e per il Pronto Soccorso comportano, a causa dei maggiori tempi assorbiti, un maggior consumo unitario di risorse rispetto all'attività ambulatoriale così come le attività radioscopiche di supporto interventistico.

Queste caratteristiche strutturali fanno venir meno una importante leva di manovra del risultato economico, tipica delle aziende private e in una certa misura utilizzabile dalle aziende sanitarie private, che è costituita dalla manovra del mix di produzione attraverso l'offerta delle sole prestazioni che consentono una adeguata remunerazione dei fattori produttivi impiegati e il potenziamento all'interno di queste delle prestazioni maggiormente remunerative.

Alcune prestazioni, inoltre, sono associate ad una tariffa che risulta, pur operando in condizioni di efficienza, scarsamente remunerativa, specie se per la loro esecuzione viene richiesta l'applicazione di particolari protocolli.

Notevole è infine il peso delle attività tecniche ed amministrative che risultano indirette rispetto alla produzione.

La conoscenza analitica dell'andamento dei costi e dei ricavi con riferimento ad un oggetto di calcolo più ristretto dell'unità operativa rimane tuttavia di fondamentale importanza ai fini della programmazione delle attività, in quanto consente di stimare a priori per volumi o mix di produzione differenti e in relazione a scenari e ipotesi diverse il risultato economico corrispondente.

\section{Figura 1}

Le determinanti della metodologia di analisi dei costi.

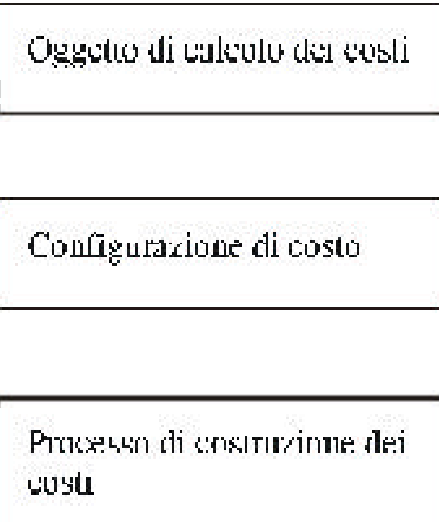

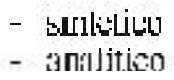

- pieno

- diretro

- răuiabilọ

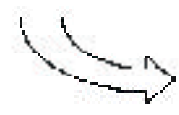

-

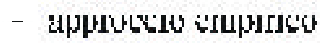


In questo quadro è importante sottolineare che, seppur limitati o condizionati, gli spazi per il miglioramento esistono: il primo passo in questa direzione consiste nell' analizzare per conoscere e misurare, dal momento che non si può migliorare ciò che non si conosce e ciò che non si misura.

\section{DISCUSSIONE}

Le metodologie di analisi dei costi

Quando si parla di analisi dei costi di una qualsiasi attività, sanitaria o di altro tipo, si devono prendere in considerazione due aspetti:

- la definizione del modello teorico di analisi;

- l'applicazione di tale modello, finalizzata a costruire il costo dell'attività presa in esame.

All'interno del modello di valutazione dei costi si possono individuare tre grossi elementi.

Il primo riguarda l'individuazione dell'oggetto di calcolo cui riferire l'analisi.

Il secondo è quello relativo alla configurazione di costo prescelta: in pratica si fa riferimento alle tipologie di costo che si devono considerare nella valutazione.

Il terzo fa riferimento al processo di costruzione del costo, inteso come l'insieme delle tecniche da utilizzare, nell'applicazione reale, per arrivare a calcolare il costo.

Approfondiamo meglio i tre aspetti partendo dall'oggetto di calcolo.

\section{L'oggetto di calcolo dei costi}

L'oggetto di calcolo è rappresentato da una "parte" dell'azienda della quale si reputa importante conoscere l' attribuzione dei costi: l'oggetto di calcolo più analitico rispetto al quale è possibile ripartire costi e ricavi è rappresentato dal singolo prodotto anche se non sempre l'impegno (in termini di informazioni da gestire e di risorse necessarie) che un oggetto di rilevazione sistematica così ristretto comporta è controbilanciato da una effettiva utilità pratica.

Gli oggetti di calcolo dei costi per un'azienda sanitaria locale possono infatti essere (dal più sintetico al più analitico):

- l'azienda sanitaria locale nel suo complesso;

- il livello essenziale di assistenza (assistenza sanitaria collettiva in ambiente di vita e di lavoro, assistenza distrettuale, assistenza ospedaliera);

- il sotto livello assistenziale;

- il singolo distretto, il singolo presidio ospedaliero;

- il centro di responsabilità o il centro di costo;

- l'utenza di riferimento (utenti residenti nell' ASL, utenti residenti in altra ASL regio- nale, utenti residenti in altre regioni);

- il singolo progetto obiettivo;

- il singolo dipartimento (ospedaliero, prevenzione, salute mentale);

- l'unità operativa (ospedaliera e territoriale);

- le attività svolte all'interno dell'unità operativa (degenza ordinaria, day hospital, ambulatorio per le unità operative cliniche ospedaliere);

- determinati raggruppamenti di prodotti o di prestazioni (chirurgiche, mediche);

- il singolo prodotto o prestazione;

ogni altro disaggregato del complesso aziendale di cui può essere utile conoscere costi e ricavi (es. assistenza prodotta e assistenza acquistata).

Nell'optare per un oggetto di calcolo dei costi e dei ricavi più o meno analitico si dovrà tenere conto dei seguenti fattori:

- l'utilità ai fini decisionali;

- l'effettiva possibilità di rilevare le informazioni;

- 1'opportunità di effettuare più aggregazioni diverse che solo oggetti di calcolo analitici possono offrire;

- l'onere di rilevazione connesso ad oggetti di calcolo estremamente analitici.

Il concetto fondamentale è che non esiste un metodo unico o migliore per impostare un calcolo analitico dei costi e dei ricavi, ma che ogni situazione d'azienda, e nel caso estremo ogni tipologia diversa di decisione, va attentamente valutata al fine di predisporre l'informazione di costo più utile.

\section{La configurazione di costo}

La configurazione identifica le tipologie di costo che si vogliono prendere in considerazione nella valutazione.

Si può prevedere di rilevare i costi di tutte le risorse che concorrono alla realizzazione dell'attività (del prodotto o dell'oggetto di cui si vuole calcolare il costo); in questo caso la configurazione sarà quella del costo pieno $o$ full cost.

Calcolare il costo pieno di un oggetto vuole dire censire i costi di tutte le risorse che sono state impiegate per la sua realizzazione. Nel caso di un prodotto industriale dovremmo includere: il costo delle materie prime, $i$ costi di produzione (macchinari, personale, energia), ma anche $i$ costi di progettazione, della vendita, i costi dell'amministrazione e così via.

Nel caso di una prestazione sanitaria, calcolare il costo pieno equivale a considerare i costi del personale, i costi dei materiali sanitari, il consumo dei farmaci, l'uso delle attrezzature, delle strutture fisiche (ambulatori, reparti di degenza, sale operatorie), nonché i costi dell'amministrazione centrale (i cosiddetti 
servizi centrali quali, Provveditorato, Economato, Direzione generale).

Diverso è invece il caso in cui si vogliano considerare solo i costi di alcune risorse e non di tutte. Queste configurazioni sono dette parziali e possono assumere varie connotazioni in funzione di quali costi si decide di inserire nel calcolo.

Possiamo includere solo costi di tipo diretto rispetto all'oggetto di calcolo, in questo caso si parla di direct cost.

Sempre con riferimento ad una prestazione sanitaria, calcolando il costo diretto dovremo considerare solo i costi oggettivamente attribuibili a questa, quali il tempo del personale, $\mathrm{i}$ materiali e i farmaci, le attrezzature specifiche impiegate per l'erogazione della prestazione, tralasciando invece i costi dell' amministrazione e dei servizi centrali.

Un altro tipo di configurazione è quella che include solo i costi variabili, detta variable cost (sono variabili i costi di quelle risorse il cui ammontare varia al modificarsi del livello di attività svolta; fissi, di contro, quelli che non si modificano al variare dei volumi di attività realizzata).

Per fare alcuni esempi, sempre rispetto alla nostra prestazione sanitaria, considereremo come costo variabile il tempo speso dai medici e da altre categorie professionali per l'erogazione della prestazione e il costo dei materiali e dei farmaci; tali costi crescono in maniera proporzionale al crescere dell'attività svolta.

Sono fissi invece i costi delle attrezzature e delle strutture, i costi dell' amministrazione e dei servizi centrali (il loro valore non si modifica erogando una prestazione aggiuntiva) e non devono pertanto essere considerati.

Così facendo abbiamo identificato tre particolari configurazioni di costo:

- il costo pieno;

- il costo diretto;

- il costo variabile.

Le tre configurazione vengono solitamente utilizzate per finalità diverse.

Il costo diretto, in quanto rappresenta il valore dei costi che verrebbe eliminato (attivato) in caso di cessazione (inizio) dell'attività, è utilizzato usualmente nelle analisi differenziali, in cui si valuta l'effetto dell'eliminazione, o al contrario, dell'avviamento di un'attività o di un'azione.

Il costo variabile invece si considera quando si vuole apprezzare l'effetto dell'aumento o diminuzione di una unità di attività o di prodotto sui costi totali. Il costo variabile è infatti anche detto costo marginale, ovvero l'ammontare di costo sostenuto per realizzare una unità aggiuntiva di output.

Infine, il costo pieno è utilizzato in particolare modo quando si vuole fare un' analisi completa di tutti i costi di un'attività (o un prodotto) finalizzata, ad esempio, a definire il prezzo di vendita o la redditività (intesa come differenza tra prezzo e costo) della stessa.

Fra i tre metodi proposti, quello che soddisfa meglio le finalità di uno studio volto a valutare l'economicità della gestione di un'unità operativa è senz'altro l'approccio del costo diretto.

\section{Il processo di costruzione del costo}

Proseguiamo ora nella nostra analisi parlando del secondo punto che caratterizza i modelli di valutazione, ovvero il processo di costruzione del costo.

Sotto questo profilo sono individuabili due ipotesi alternative:

- il metodo teorico;

- il metodo empirico.

I due approcci si differenziano in funzione delle diverse modalità di costruzione del costo adottate.

L'approccio teorico si basa sulla definizione del costo di un oggetto (ad esempio una prestazione sanitaria), attraverso l'identificazione a tavolino delle risorse necessarie per realizzarlo. In questo caso l'efficienza di utilizzo, ovvero il livello di risorse impiegate per unità prodotta, dipenderà dalle ipotesi assunte dall'analista. Ci si può tarare, ad esempio, su un livello ottimale di consumo basato su un riferimento di eccellenza (best practice) oppure su un livello normale di utilizzo riferito ad una gestione "ordinaria".

Sempre in relazione ad una prestazione, e nell'ipotesi di considerare come configurazione di costo il full cost, applicare il metodo teorico consisterebbe nella ricostruzione a tavolino di tutti gli impieghi di risorse necessarie per erogare la prestazione (personale, farmaci, materiali, strutture, ecc.).

Il metodo empirico si basa invece su una costruzione del costo, e quindi dell'utilizzo delle risorse, basato sull'osservazione della realtà. Sempre nel caso della prestazione sanitaria, ricavare il costo empiricamente equivarrebbe a "osservare" l'erogazione della prestazione per un predefinito arco di tempo, misurando gli utilizzi di risorse necessari per la sua realizzazione: il tempo effettivamente impiegato, i materiali e i farmaci realmente utilizzati, l'impegno della struttura (in termini di giornate) effettivamente sostenuto.

Questa osservazione può essere svolta nel continuo e in questo caso siamo in presenza di modelli di rilevazione e controllo routinari, oppure una tantum in predeterminati momenti.

Ovviamente tra le due, l'osservazione continua è da preferirsi per il fatto che consente di seguire e monitorare il "fenomeno" momento per momento. Indubbiamente in alcuni casi 
questo non è possibile ${ }^{\mathrm{i}} \mathrm{e}$, come spesso accade nell' attività sanitaria, ci si affida a osservazioni puntuali.

Nel caso di rilevazioni di quest'ultimo tipo, occorre scegliere la "finestra" di osservazione in modo tale da garantire alla stessa la massima significatività di risultato.

In particolare occorre definire la durata (per quanto tempo) e il periodo (quando) dell'osservazione.

In merito alla durata possiamo dire che questa dovrebbe mantenersi tra due limiti.

Da una parte, deve essere abbastanza ampia da consentire di analizzare il fenomeno per un lasso di tempo sufficiente ad avere un campione di dati significativo. Nel caso di rilevazione del costo di prestazione una durata ottimale è quella che garantisce la possibilità di rilevare un numero ragguardevole di prestazioni.

Dall'altra, non deve essere troppo lunga in modo da non intaccare l'attendibilità della misurazione. Questo aspetto può sembrare per certi versi paradossale, in realtà se pensiamo alle attività e alle condizioni in cui è applicato il metodo della rilevazione una tantum, riusciremo a comprenderlo meglio. Questa modalità è infatti utilizzata in situazioni particolari in cui i costi di analisi sono molto onerosi (pensiamo alla valutazione dei carichi di lavoro) o in contesti che, per scarsa "cultura della misurazione", non sono pronti ad accettare rilevazioni continue. In questi ed analoghi casi, prolungare la durata dell'osservazione per troppo tempo può avere come conseguenza la diminuzione dell'attenzione e della precisione degli operatori nella rilevazione.

Relativamente alla scelta del periodo possiamo dire che questa è tanto cruciale quanto più l'attività da osservare ha delle fluttuazioni o ciclicità di intensità (stagionali, mensili). In questo caso occorrerebbe posizionare il periodo in modo tale da non interferire con le fasi di ipo o iperattività che porterebbero a dei dati non rispondenti al complessivo andamento del fenomeno.

Ritornando al discorso generale sulle due modalità di costruzione dei costi possiamo tentare di individuare pregi e difetti di entrambi.

In particolare l'approccio teorico annovera nel conto dei pregi:

- la più facile operatività, in termini di minore impegno di costruzione del costo;

- la possibilità di ragionare su livelli di efficienza ottimali, slegati quindi dal contesto contingente.

I limiti sono direttamente collegati ai pregi e sono connotabili in termini di ridotto legame dei valori ricavati con la realtà del fenomeno.

Passando in rassegna le caratteristiche dell' approccio empirico possiamo segnalare come principale pregio la possibilità di fotografare la realtà in modo fedele; dall'altro lato della bilancia sono peraltro da considerare i maggiori costi di applicazione e la sua forte dipendenza dalla motivazione e dalla precisione degli operatori che effettuano la rilevazione.

Alla resa dei conti non vi è un approccio nettamente superiore e preferibile rispetto all'altro. A far propendere la scelta su uno dei due saranno di volta in volta la tipologia di attività da osservare, il contesto in cui si effettua la misurazione, il tempo e le risorse a disposizione, le finalità che si perseguono con la rilevazione e così via.

Una giusta sintesi tra i due può essere trovata, qualora il tempo e le risorse a disposizione lo permettano, applicando entrambi i metodi. Tale strada è stata in parte seguita nella ricerca sui costi dell'unità operativa di diagnostica per immagini.

Il modello di valutazione dei costi per l'unità operativa di diagnostica per immagini

Come evidenziato nel paragrafo precedente, la scelta del modello di analisi dei costi di un'attività deve essere condotta tenendo in considerazione una molteplicità di fattori.

La definizione del modello di analisi dei co-

\section{Nota $\mathbf{i}$}

Nel valutare la scelta tra le due modalità di osservazione bisogna considerare vari fattori, tra $i$ quali:

- i costi dell'osservazione, da confrontare con i benefici; - la tipologia di attività o di fenomeno da osservare; la sua dinamicità nel tempo.
Fregi Lifeti

Approciolo lionico

$$
\begin{gathered}
\text { Temepi costi di } \\
\text { realiz<azione contenuti } \\
\text { ossubulitì di detinire cost } \\
\text { standard }
\end{gathered}
$$

App 3 roccio entpirico
Tempi e costi di rallizzmzons sorat

Rischio di scarsa sidutitjegtivila della rileracione
Figura 2

Approccio teorico ed empirico: pregie difetti. 
sti dell'attività radiologica non si è sottratta a questa buona regola e si è basata su valutazioni e considerazioni particolari.

Di seguito illustreremo le scelte fatte in termini di oggetto di calcolo, configurazione di costo e di modello di costruzione dei costi, indicando anche $\mathrm{i}$ fattori che hanno portato alla loro identificazione.

L'oggetto di calcolo sul quale fondare l' analisi è stato individuato nel singolo prodotto. Dal momento che l'obiettivo primario dello studio era costituito dall'individuazione delle cause di un risultato non soddisfacente dal punto di vista economico, la strada da percorrere passava necessariamente dalla scomposizione del risultato complessivo dell'unità operativa, nel risultato di ciascuna famiglia di prestazioni, e del risultato di ciascuna di queste tra tutti le prestazioni (o prodotti) che concorrono a determinarlo.

Per fare questo occorreva da un lato individuare i ricavi per ogni singolo prodotto, dall'altro procedere all'attribuzione dei costi al prodotto: ma quanti costi ripartire? Tutti i costi o solo quei costi che risultavano oggettivamente attribuibili a tale oggetto di calcolo?

La configurazione di costi che è stata ritenuta più adatta rispetto alle finalità dell' analisi è quella a costi diretti che prevede l'attribuzione all'oggetto dei costi diretti indipendentemente dal fatto che questi siano variabili (consumi di beni sanitari) o fissi (costo degli ammortamenti delle attrezzature e dei macchinari): secondo questa configurazione non viene imputata invece agli oggetti di calcolo la quota di costi fissi indiretti che viene considerata nella sua globalità.

Per le decisioni di gestione operativa riguardanti il breve termine (con questa definizione si intendono le scelte correnti che non modificano la capacità produttiva e la struttura dei costi fissi), la configurazione dicosto più appropriata è quella di costo variabile. I costi fissi, pur essendo presenti, sono in questi casi irrilevanti in quanto non essendo possibile nel breve periodo modificare la capacità produttiva non sono in grado di influire sulle decisioni di convenienza economica.

Spostandosi invece verso un'ottica di lungo periodo i costi fissi, e in particolare quelli diretti, acquistano rilevanza con riferimento alle decisioni strategiche cioè che comportano modifiche alle strutture produttive (ad es. acquistare un nuovo macchinario o allestire un nuovo ambulatorio).

Inoltre il calcolo a costi diretti pone maggiore enfasi sulle aree gestionali, sulle attività e soprattutto sui centri di responsabilità, migliorando la conoscenza dei responsabili di tutti i costi di pertinenza dell' area da essi controllata, e mettendoli quindi in grado di agire su di essi.

Occorre considerare ora il terzo elemento, ovvero la modalità di costruzione del costo.

Come evidenziato precedentemente, nello scegliere la modalità di costruzione del costo per l'analisi dell'unità operativa di diagnostica per immagini sarebbe stato possibile muoversi tra due alternative diverse.

Da una parte un criterio teorico, consistente nella costruzione a tavolino dei consumi di risorse necessari per erogare ogni prestazione; dall'altra, un approccio empirico di rilevazione "sul campo" dei costi sostenuti realmente.

Il criterio di costruzione del costo applicato nella ricerca è un ibrido tra i due approcci appena menzionati. Infatti si è utilizzato in larga parte l'approccio teorico sul quale è stata fatta qualche ritaratura di carattere empirico.

La fase teorica è consistita nella definizione di modalità di erogazione standard, in termini di:

- tempi diretti di prestazione;

- composizione dell'équipe (medico, tecnico e infermiere);

- consumi di farmaci e materiali;

- utilizzo di attrezzature e macchine;

- verifiche di congruità tra la somma dei valori standard ed i valori effettivi.

È importante sottolineare che lo standard desunto dall'elaborazione teorica non è da intendersi come modalità "ottimale" di erogazione, in quanto deriva da una scelta fatta sulla base di dati reali di erogazione. È piuttosto uno standard di "normale" efficienza, espressione quindi di una "ordinaria" configurazione di erogazione (in termini di composizione di équipe e di tempo dedicato).

La fase empirica si è articolata nella verifica mediante una serie di rilevazioni sul campo aventi lo scopo di validare gli standard individuati.

Gli standard di prestazione identificati non costituivano peraltro ancora dei valori di costo, ma solo dei tempi di personale dedicato alla prestazione.

Per tradurli in costo è stata necessaria una valorizzazione dei medesimi mediante l'utilizzo di costi standard e di costi effettivi: attraverso tale valorizzazione si è ottenuto il costo diretto di prestazione che misura il valore economico delle risorse dirette consumate.

I risultati dello studio, sotto il profilo dell'analisi dei costi e delle tariffe, sono illustrati nei paragrafi che seguono.

\section{Le fasi dello studio}

La fase preliminare è stata rappresentata dalla raccolta dei valori economici da utilizzare per l'analisi. A tal fine, grazie anche alla presenza di una contabilità analitica di presidio evoluta ed attendibile, sono stati utilizzati come dati 

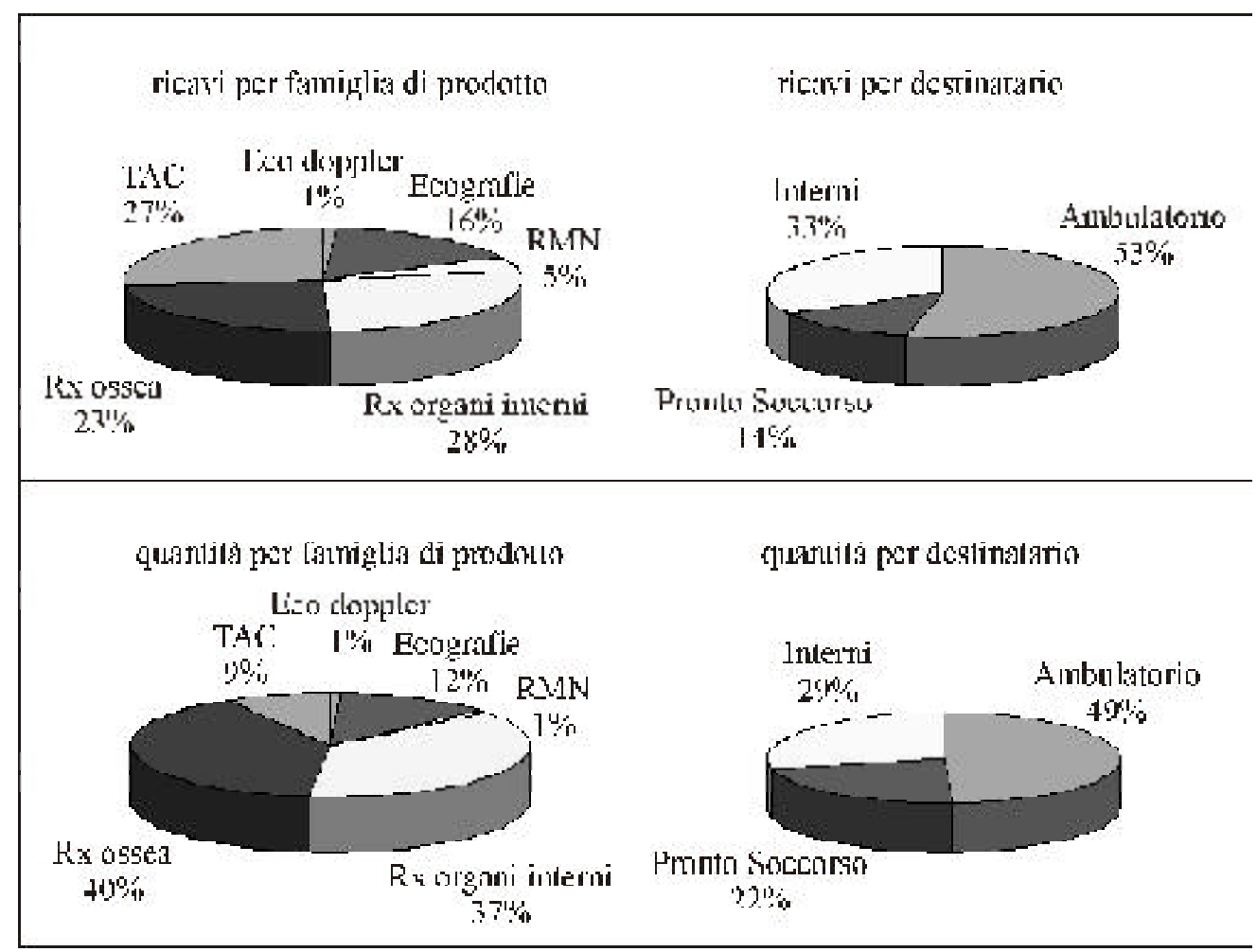

Figura 3

Mix di produzione.

storici di partenza i valori economici riferiti all'anno precedente, in quanto ritenuti completi ed assestati, relativi alle quantità prodotte, ai ricavi, e soprattutto ai costi diretti dell'unità operativa.

La metodologia seguita ha comportato le seguenti fasi di lavoro:

1) Raggruppamento dei prodotti in famiglie di prodotti;

2) Attribuzione ai prodotti di quantità e ricavi;

3) Ordinamento dei prodotti all'interno dei raggruppamenti per quantità decrescenti;

4) Rilevazione del consumo unitario standard di risorse per prodotto;

5) Determinazione dei costi unitari standard per prodotto mediante quantificazione monetaria dei consumi;

6) Determinazione delle ore totali che sarebbero state necessarie in base ai tempi standard e raffronto con le ore totali effettivamente prestate dal personale;

7) Determinazione del margine unitario per prodotto e del margine totale sulla base delle quantità prodotte e delle percentuali di incidenza;

8) Considerazioni sulla redditività dei prodotti e delle famiglie di prodotti;

9) Attribuzione ai prodotti degli ammortamenti calcolati in base al costo di sostituzione e al periodo di vita utile delle macchine;

10) Ipotesi di miglioramento e simulazione delle possibili conseguenze economiche.

\section{1) Raggruppamento dei prodotti in famiglie} di prodotti

I singoli esami o prodotti del reparto Radiologia sono stati attribuiti alle seguenti aggregazioni significative:

a) Eco Doppler;

b) Ecografia;

c) Risonanza Magnetica;

d) RX Organi interni;

e) RX Ossea;

f) $\mathrm{TAC}$

2) Attribuzione ai prodotti di quantità e ricavi

Ad ogni singolo prodotto sono state quindi attribuite le quantità prodotte nell' anno precedente con distinzione delle quantità prodotte per esterni, per interni e per il Pronto Soccorso.

Al termine del raggruppamento e dell'attribuzione di quantità e ricavi è stato possibile avere un quadro completo dell'attività dell'unità operativa sia per quanto concerne la composizione dei ricavi per famiglia di prodotto e per destinatario sia per quanto concerne la distribuzione delle quantità, come evidenziato nella Figura 3 -Mix di produzione-.

\section{3) Ordinamento dei prodotti all'interno dei} raggruppamenti per quantità decrescenti

Questa fase ha avuto lo scopo di mettere in evidenza i prodotti che all'interno di ogni famiglia hanno avuto maggior peso nel mix delle quantità prodotte al fine di concentrare l'atten- 
zione, in prima analisi, sulle prestazioni più frequentemente eseguite.

4) Rilevazione del consumo unitario standard di risorse per prodotto

Tale fase ha riguardato la rilevazione di:

a) minuti esecuzione medico;

b) minuti esecuzione tecnico;

c) minuti esecuzione infermiere;

d) minuti esecuzione consulente;

e) minuti refertazione medico;

f) numero e tipo di immagini e pellicole radiologiche;

g) quantità eventuale mezzo di contrasto;

h) eventuale altro materiale diretto (siringa, catetere, gel).

5) Determinazione dei costi unitari standard per prodotto mediante quantificazione monetaria dei consumi effettuata come segue:

- tempo del medico radiologo 104.000 lire/ora in base al rapporto tra costo e ore dell' anno precedente;

- tempo di tecnico sanitario di radiologia medica e dell'infermiere professionale 47.000 lire/ora in base al rapporto tra costo e ore dell'anno precedente;

- tempo del consulente esterno utilizzato per le prestazioni di risonanza magnetica 300.000 ora in base alle specifiche convenzioni;

- immagini e pellicole: stima del costo per ogni prodotto sulla base delle quantità consumate;

- mezzo di contrasto: stima del costo per ogni prodotto sulla base delle quantità consumate;

- eventuale altro materiale diretto (siringa, catetere, gel): stima del costo per ogni prodotto sulla base delle quantità consumate.

6) Determinazione delle ore totali che sarebbero state necessarie in base ai tempi standard e raffronto con le ore totali effettivamente prestate dal personal

Tale confronto è stato effettuato allo scopo di individuare per medici e tecnici/infermieri eventuali differenze da imputarsi a:

- sopra o sottovalutazione dei tempi necessari;

- maggiore o minore efficienza.

Stando ai tempi standard rilevati e mettendoli in relazione con le ore effettivamente prestate da medici e tecnici si è rilevato quanto segue.

I medici hanno impiegato complessivamente meno ore di quelle che risultano dalla somma dei tempi standard moltiplicati per le attività svolte:

- tempo impiegato complessivamente nell' anno di riferimento: 11.804 ore, pari a 100;

- tempo che in base agli standard sarebbe stato necessario: 13.047 ore, pari a 111.
Tale fatto è riconducibile ad una maggior efficienza rispetto agli standard dell' attività medici pari al 10\% [(13.047 - 11.804) / 11804].

I tecnici hanno impiegato complessivamente più ore di quelle che risultano dalla somma dei tempi standard moltiplicati per le attività svolte:

- tempo impiegato complessivamente nell'anno: 19.963 ore, pari a 100 ,

- tempo che in base agli standard sarebbe stato necessario: 10.423 ore, pari a 52 .

Tale fatto è riconducibile ad una inefficienza nell'impiego dei tecnici in attività di produzione diretta pari al 48\% [(10.423 - 19.963) / 19.963] che può essere riconducibile alle seguenti ragioni:

attività prestata da tecnici quali addetti agli amplificatori di brillanza in sala operatoria di Chirurgia o di Ortopedia per interventi eseguiti in radioscopia (attività aggiuntiva rispetto agli esami diagnostici);

attività prestata da tecnici, quali addetti agli amplificatori di brillanza, per i trattamenti di Terapia del Dolore (attività aggiuntiva rispetto agli esami diagnostici);

esami diagnostici eseguiti al letto (ad esempio Rx torace su Infartuato) o esami di radiologia ossea eseguiti in sala risveglio immediatamente dopo interventi ortopedici, con tempi maggiori dei tempi standard;

presenza nell' anno oggetto di analisi di tecnici appena diplomati da formare mediante affiancamento e quindi non direttamente produttivi;

presenza di personale tecnico inidoneo ai raggi $X$ che svolge attività indiretta;

saltuario impiego dei tecnici in altre attività indirette rispetto alla produzione;

presenza di un Capo Tecnico di Radiologia che svolge principalmente attività indirette (coordinamento, ecc).

7) Determinazione del margine unitario per prodotto e del margine totale sulla base delle quantità prodotte e delle percentuali di incidenza

I ricavi che sono stati imputati ad ogni singolo prodotto mediante la metodologia utilizzata rappresentano la totalità dei ricavi dell' anno oggetto di analisi del reparto Radiologia pari a circa 3.106 miliardi.

I costi diretti di reparto che sono stati complessivamente attribuiti ad ogni singolo prodotto, ai fini del calcolo del margine, ammontano rispettivamente al:

$65 \%$ dei costi di Beni e Servizi sanitari e non sanitari, pari a 1.029 milioni su un valore totale di 1.578;

- $76 \%$ dei costi relativi al Personale pari a 1.929 milioni su un valore totale di 2.549 , per un totale complessivo di 2.958 milioni 


\begin{tabular}{|lccccc}
\hline \multicolumn{1}{|c}{ VOCI } & Totale & $\begin{array}{c}\text { Attribuiti } \\
\text { a livello } \\
\text { prodotto }\end{array}$ & $\begin{array}{c}\text { \% } \\
\text { attribuiti }\end{array}$ & $\begin{array}{c}\text { Non } \\
\text { attribuiti } \\
\text { al livello } \\
\text { prodotto }\end{array}$ & $\begin{array}{c}\text { \% non } \\
\text { attribuiti }\end{array}$ \\
\hline \hline Ricavi & 3.106 & 3.106 & $100 \%$ & & \\
\hline \hline $\begin{array}{l}\text { Costo beni e sevizi } \\
\text { sanitari e non sanitari }\end{array}$ & -1.578 & -1.029 & $65 \%$ & -549 & $35 \%$ \\
\hline Costo del personale & -2.549 & -1.929 & $76 \%$ & -620 & $24 \%$ \\
\hline Altri costi della produzione & -237 & & & -237 & $100 \%$ \\
\hline Totale Costi & -4.364 & -2.958 & $68 \%$ & -1.406 & $32 \%$ \\
\hline Risurta & & & &
\end{tabular}

Tabella 1

Costi diretti di unità operativa attribuiti a livello di prodotto. pari a circa il $68 \%$ dei costi totali di reparto.

I costi diretti che non sono stati attribuiti ad ogni singolo prodotto, e che restano quindi esclusi dal calcolo del margine di prodotto, ammontano quindi per differenza al:

- $35 \%$ dei costi relativi a Beni e Servizi sanitari e non sanitari, pari a 549 milioni su un totale di 1.578;

- $24 \%$ dei costi relativi al Personale pari a 620 milioni su un totale annuo di 2.549, pari al costo del personale amministrativo diretto di unità operativa ma indiretto rispetto al singolo prodotto e del personale tecnico che svolge attività indirette di produzione;

- $\quad 100 \%$ degli altri costi della produzione pari a 237 milioni, in quanto essendo relativi a riscaldamento, pulizia, manutenzione dello stabile ecc. seppur considerabili come costi diretti di unità operativa risultano essere indiretti rispetto ai prodotti.

Per un totale complessivo di 1.406 milioni pari a circa il $32 \%$ dei costi totali di unità operativa.

Mediante l'analisi così effettuata è stato possibile determinare:

- il margine di contribuzione unitario e percentuale relativo ad ogni singolo prodotto, determinato come differenza tra la tariffa relativa allo specifico esame ed il suo costo diretto unitario;

- il margine di contribuzione complessivo generato dal singolo prodotto, ottenuto moltiplicando il margine unitario per le quantità prodotte nell'anno.

Nella Tabella 2 -Prodotti ordinati per margine unitario decrescente- sono riportati tutti i prodotti ordinati, indipendentemente dalla famiglia di appartenenza, a partire da quello col miglior margine unitario in termini assoluti per finire con il prodotto col peggior margine unitario in valore assoluto.

8) Considerazioni sulla redditività di prodotto e di famiglia di prodotto

Considerazioni generali

L'attribuzione a livello di singolo prodotto di tutti i ricavi (3.100 milioni) e del 68\% dei costi diretti di reparto (2.958 milioni) ha permesso di determinare il valore del margine complessivo di prodotto e di famiglia di prodotti. Tale margine esprime il potenziale di contribuzione di ciascun prodotto alla copertura dei rimanenti costi di unità operativa (1.406 milioni) e della quota di costi generali di azienda.

Nella Tabella 3 - Conto economico di sintesi- è riportato il conto economico di sintesi nel quale sono riportati i valori unitari medi per famiglia di prodotto e i valori totali per famiglia di prodotto relativi all' anno di riferimento, mentre la Figura 4 - Ricavi, costi diretti e margini complessivi per famiglia di prodotto- permette di apprezzarne graficamente le differenze.

È abbastanza evidente, e ciò conferma l'utilità di aver optato per un oggetto di calcolo estremamente analitico quale la singola prestazione, che esistono sostanziali differenze a livello unitario tra prodotti diversi appartenenti alla stessa famiglia. Tali differenze sono ovviamente amplificate, a livello totale, dalle quantità prodotte per ogni esame nel corso dell'anno per cui possiamo notare, dal punto di vista economico, i seguenti comportamenti estremi:

prestazioni con un leggero margine unitario negativo che producono un margine totale pesantemente negativo in quanto prodotti in quantità molto elevate;

prestazioni con un margine unitario fortemente negativo che producono un margine totale negativo modesto in quanto prodotti in quantità limitate;

prestazioni con un leggero margine unitario positivo che producono un margine totale positivo e consistente in quanto prodotti in quantità molto elevate;

prestazioni con un forte margine unitario positivo che producono un margine totale positivo modesto in quanto prodotti in quantità limitate.

Le regole di una gestione orientata alla redditività (ovviamente in assenza di vincoli sulla tipologia e la quantità di esami offerta) 


\begin{tabular}{|c|c|c|c|c|c|c|c|}
\hline \\
\hline & Raggruppamento & Descrizione & $\begin{array}{r}\text { Tariffa } \\
\text { unitaria }\end{array}$ & $\begin{array}{l}\text { Costo } \\
\text { diretto } \\
\text { unitario }\end{array}$ & $\begin{array}{c}\text { Margine } \\
\text { unitario } \\
\text { in lire }\end{array}$ & $\begin{array}{c}\text { Margine } \\
\% \text { sulla } \\
\text { tariffa }\end{array}$ & $\begin{array}{c}\text { Mix sul } \\
\text { totale } \\
\text { ricavi }\end{array}$ \\
\hline & TAC & $\begin{array}{l}\text { Tomografia computerizzata (tc) } \\
\text { dell' addome completo, senza }\end{array}$ & 340.000 & 80.583 & 259.417 & $76 \%$ & $1.18 \%$ \\
\hline & TAC & $\begin{array}{l}\text { Tomografia computerizzata (tc) } \\
\text { dell' addome completo }\end{array}$ & 223.000 & 93.167 & 129.833 & $58 \%$ & $0.07 \%$ \\
\hline & TAC & $\begin{array}{l}\text { Tomografia computerizzata (tc) } \\
\text { dell' arto superiore }\end{array}$ & 176.000 & 60.750 & 115.250 & $65 \%$ & $0.05 \%$ \\
\hline & TAC & $\begin{array}{l}\text { Tomografia computerizzata (tc) } \\
\text { del capo }\end{array}$ & 161.000 & 47.400 & 113.600 & $71 \%$ & $4.52 \%$ \\
\hline & TAC & Densitometria ossea con tc & 149.000 & 41.083 & 107.917 & $72 \%$ & $1.90 \%$ \\
\hline & TAC & $\begin{array}{l}\text { Tomografia computerizzata (tc) } \\
\text { del bacino }\end{array}$ & 167.500 & 60.750 & 106.750 & $64 \%$ & $0.03 \%$ \\
\hline & TAC & $\begin{array}{l}\text { Tomografia computerizzata (tc) } \\
\text { dei reni, senza e con contras }\end{array}$ & 289.000 & 183.917 & 105.083 & $36 \%$ & $0.15 \%$ \\
\hline & TAC & $\begin{array}{l}\text { Tomografia computerizzata (tc) } \\
\text { dell' addome superiore }\end{array}$ & 171.000 & 73.333 & 97.667 & $57 \%$ & $0.41 \%$ \\
\hline & TAC & $\begin{array}{l}\text { Tomografia computerizzata (tc) } \\
\text { dell' addome inferiore }\end{array}$ & 171.000 & 73.333 & 97.667 & $57 \%$ & $0.04 \%$ \\
\hline & TAC & $\begin{array}{l}\text { Tomografia computerizzata (tc) } \\
\text { del collo }\end{array}$ & 161.000 & 66.667 & 94.333 & $59 \%$ & $0.16 \%$ \\
\hline & TAC & $\begin{array}{l}\text { Tomografia computerizzata (tc) } \\
\text { dell' arto inferiore }\end{array}$ & 167.500 & 76.667 & 90.833 & $54 \%$ & $0.79 \%$ \\
\hline & TAC & $\begin{array}{l}\text { Tomografia computerizzata (tc) } \\
\text { del massiccio facciale }\end{array}$ & 173.000 & 84.500 & 88.500 & $51 \%$ & $0.70 \%$ \\
\hline & TAC & $\begin{array}{l}\text { Tomografia computerizzata (tc) } \\
\text { del capo, senza e con contras }\end{array}$ & 259.000 & 184.533 & 74.467 & $29 \%$ & $1.99 \%$ \\
\hline & RMN & $\begin{array}{l}\text { Risonanza magnetica nucleare (rm) } \\
\text { muscoloscheletrica }\end{array}$ & 344.000 & 275.833 & 68.167 & $20 \%$ & $5.09 \%$ \\
\hline & $\mathrm{Rx}$ organi intemi & $\begin{array}{l}\text { Radiografia completa del tubo } \\
\text { digerente }\end{array}$ & 136.500 & 70.083 & 66.417 & $49 \%$ & $0.33 \%$ \\
\hline & TAC & $\begin{array}{l}\text { Tomografia computerizzata (tc) } \\
\text { del rachide e dello speco ve }\end{array}$ & 167.000 & 102.333 & 64.667 & $39 \%$ & $2.87 \%$ \\
\hline & Rx organi interni & Isterosalpingografia & 210.000 & 147.833 & 62.167 & $30 \%$ & $0.06 \%$ \\
\hline & TAC & $\begin{array}{l}\text { Tomografia computerizzata (tc) } \\
\text { dell' orecchio, senza e con c }\end{array}$ & 259.000 & 202.583 & 56.417 & $22 \%$ & $0.12 \%$ \\
\hline & TAC & $\begin{array}{l}\text { Tomografia computerizzata (tc) } \\
\text { dell' orecchio }\end{array}$ & 161.000 & 104.917 & 56.083 & $35 \%$ & $0.27 \%$ \\
\hline & Ecografia & $\begin{array}{l}\text { Biopsia [percutanea] [agobiopsia] } \\
\text { della tiroide }\end{array}$ & 119.000 & 69.200 & 49.800 & $42 \%$ & $0.06 \%$ \\
\hline & Ecografia & Ecografia addome completo & 117.000 & 69.200 & 47.800 & $41 \%$ & $4.72 \%$ \\
\hline & TAC & $\begin{array}{l}\text { Tomografia computerizzata (tc) } \\
\text { del torace }\end{array}$ & 167.000 & 120.000 & 47.000 & $28 \%$ & $1.01 \%$ \\
\hline & Ecografia & Ecografia dell' addome superiore & 85.000 & 59.133 & 25.867 & $30 \%$ & $4.45 \%$ \\
\hline & Ecografia & Ecografia transrettale & 84.000 & 59.133 & 24.867 & $30 \%$ & $0.82 \%$ \\
\hline & Ecografia & Biopsia eco-guidata dei tessuti molli & 91.800 & 69.200 & 22.600 & $25 \%$ & $0.03 \%$ \\
\hline & TAC & $\begin{array}{l}\text { Tomografia computerizzata (tc) } \\
\text { del rachide e dello speco ver }\end{array}$ & 57.000 & 37.167 & 19.833 & $35 \%$ & $0.61 \%$ \\
\hline & EcoDoppler & $\begin{array}{l}\text { Eco(color)doppler dei tronchi } \\
\text { sovraaortici }\end{array}$ & 85.000 & 65.300 & 19.700 & $23 \%$ & $0.12 \%$ \\
\hline & Rx organi interni & Telecuore con esofago baritato & 61.000 & 45.400 & 15.600 & $26 \%$ & $0.96 \%$ \\
\hline Tabella 2 & Rx ossea & Radiografia assiale della rotula & 51.000 & 38.600 & 12.400 & $24 \%$ & $0.14 \%$ \\
\hline $\begin{array}{l}\text { Proaottl orainat } \\
\text { margine unitari }\end{array}$ & Ecografia & Ecografia della mammella & 69.500 & 59.133 & 10.367 & $15 \%$ & $1.49 \%$ \\
\hline crescente. & Rx ossea & Radiografia completa della colonna & 67.000 & 56.667 & 10.333 & $15 \%$ & $0.78 \%$ \\
\hline
\end{tabular}




\begin{tabular}{|c|c|c|c|c|c|c|}
\hline Raggruppamento & Descrizione & $\begin{array}{r}\text { Tariffa } \\
\text { unitaria }\end{array}$ & $\begin{array}{c}\text { Costo } \\
\text { diretto } \\
\text { unitario }\end{array}$ & $\begin{array}{c}\text { Margine } \\
\text { unitario } \\
\text { in lire }\end{array}$ & $\begin{array}{c}\text { Margine } \\
\text { \% sulla } \\
\text { tariffa }\end{array}$ & $\begin{array}{c}\text { Mix sul } \\
\text { totale } \\
\text { ricavi }\end{array}$ \\
\hline Rx ossea & $\begin{array}{l}\text { Radiografia di coste, sterno e } \\
\text { clavicola }\end{array}$ & 47.000 & 38.600 & 8.400 & $18 \%$ & $1.34 \%$ \\
\hline Rx ossea & $\begin{array}{l}\text { Radiografia del cranio e dei seni } \\
\text { paranasali }\end{array}$ & 43.000 & 38.600 & 4.400 & $10 \%$ & $1.29 \%$ \\
\hline Ecografia & Ecografia osteoarticolare & 63.000 & 59.133 & 3.867 & $6 \%$ & $1.15 \%$ \\
\hline Ecografia & Ecografia dell' addome inferiore & 62.000 & 59.133 & 2.867 & $5 \%$ & $1.41 \%$ \\
\hline Ecografia & Ecografia di grossi vasi addominali & 62.000 & 59.133 & 2.867 & $5 \%$ & $0.05 \%$ \\
\hline Rx organi intemi & Tomografia [stratigrafia] renale & 60.000 & 58.200 & 1.800 & $3 \%$ & $0.72 \%$ \\
\hline Rx organi interni & Radiografia dell' apparato urinario & 37.500 & 35.733 & 1.767 & $5 \%$ & $0.98 \%$ \\
\hline Rx ossea & $\begin{array}{l}\text { Ortopanoramica delle arcate } \\
\text { dentarie }\end{array}$ & 40.000 & 38.600 & 1.400 & $4 \%$ & $1.73 \%$ \\
\hline Ecografia & Ecografia dei testicoli & 60.000 & 59.133 & 867 & $1 \%$ & $0.18 \%$ \\
\hline Rx organi interni & Radiografia del torace di routine, nas & 30.000 & 29.133 & 867 & $3 \%$ & $12.31 \%$ \\
\hline Rx organi interni & Pielografia retrograda monolaterale & 33.000 & 132.833 & 167 & $0 \%$ & $0.04 \%$ \\
\hline Rx organi interni & $\begin{array}{l}\text { Radiografia del tratto } \\
\text { gastrointestinale inferiore }\end{array}$ & 58.000 & 59.467 & $(1.467)$ & $-3 \%$ & $0.12 \%$ \\
\hline Rx ossea & Radiografia della colonna cervicale & 35.000 & 38.600 & $(3.600)$ & $-10 \%$ & $2.08 \%$ \\
\hline Rx ossea & Radiografia del piede e della caviglia & 34.500 & 38.600 & $(4.100)$ & $-12 \%$ & $2.33 \%$ \\
\hline Ecografia & Ecografia muscolotendinea & 55.000 & 59.133 & (4.133) & $-8 \%$ & $0.31 \%$ \\
\hline Ecografia & $\begin{array}{l}\text { Ecografia della cute e del tessuto } \\
\text { sottocutaneo }\end{array}$ & 55.000 & 59.133 & (4.133) & $-8 \%$ & $0.36 \%$ \\
\hline Ecografia & $\begin{array}{l}\text { Diagnostica ecografica del capo e } \\
\text { del collo }\end{array}$ & 55.000 & 59.133 & (4.133) & $-8 \%$ & $1.08 \%$ \\
\hline Rx ossea & Radiografia di pelvi e anca & 34.000 & 38.600 & $(4.600)$ & $-14 \%$ & $2.79 \%$ \\
\hline Rx organi interni & Cistouretrografia retrograda & 128.000 & 132.833 & (4.833) & $-4 \%$ & $0.02 \%$ \\
\hline Rx ossea & $\begin{array}{l}\text { Radiografia della colonna toracica } \\
\text { (dorsale) }\end{array}$ & 33.500 & 38.600 & $(5.100)$ & $-15 \%$ & $0.68 \%$ \\
\hline Rx ossea & $\begin{array}{l}\text { Radiografia della colonna } \\
\text { lombosacrale }\end{array}$ & 33.500 & 38.600 & $(5.100)$ & $-15 \%$ & $2.18 \%$ \\
\hline Rx ossea & $\begin{array}{l}\text { Radiografia completa degli arti } \\
\text { inferiori e del bacino sotto }\end{array}$ & 58.500 & 64.500 & $(6.000)$ & $-10 \%$ & $0.09 \%$ \\
\hline Rx organi interni & Radiografia dell' addome & 37.500 & 44.733 & (7.233) & $-19 \%$ & $1.25 \%$ \\
\hline Rx ossea & Radiografia della sella turcica & 29.500 & 38.600 & $(9.100)$ & $-31 \%$ & $0.01 \%$ \\
\hline Ecografia & Ecografia ginecologica & 60.000 & 69.200 & $(9.200)$ & $-15 \%$ & $0.01 \%$ \\
\hline Ecografia & Ecografia del pene & 60.000 & 69.200 & $(9.200)$ & $-15 \%$ & $0.01 \%$ \\
\hline Rx ossea & Altra radiografia di ossa della faccia & 29.000 & 38.600 & $(9.600)$ & $-33 \%$ & $0.38 \%$ \\
\hline Rx ossea & $\begin{array}{l}\text { Radiografia del gomito e dell' } \\
\text { avambraccio }\end{array}$ & 29.000 & 38.600 & $(9.600)$ & $-33 \%$ & $0.59 \%$ \\
\hline Rx organi interni & Mammografia bilaterale & 67.500 & 77.667 & $(10.167)$ & $-15 \%$ & $3.76 \%$ \\
\hline TAC & $\begin{array}{l}\text { Tomografia computerizzata (tc) } \\
\text { del massiccio facciale, senza }\end{array}$ & 275.000 & 285.500 & $(10.500)$ & $-4 \%$ & $0.23 \%$ \\
\hline Rx organi interni & $\begin{array}{l}\text { Tadiografia del tratto } \\
\text { gastrointestinale superiore }\end{array}$ & 97.000 & 107.667 & $(10.667)$ & $-11 \%$ & $0.44 \%$ \\
\hline Rx ossea & Radiografia del polso e della mano & 27.500 & 38.600 & $(11.100)$ & $-40 \%$ & $2.18 \%$ \\
\hline Ecografia & $\begin{array}{l}\text { Es. istocitopatologico mammella: } \\
\text { biopsia stereotassica }\end{array}$ & 90.000 & 102.367 & $(12.367)$ & $-14 \%$ & $0.03 \%$ \\
\hline Ecografia & Biopsia eco-guidata della mammella & 72.000 & 84.783 & $(12.783)$ & $-18 \%$ & $0.12 \%$ \\
\hline Rx ossea & Studio dell' eta' ossea & 23.500 & 38.600 & $(15.100)$ & $-64 \%$ & $0.01 \%$ \\
\hline
\end{tabular}

Segue Tabella 2. 


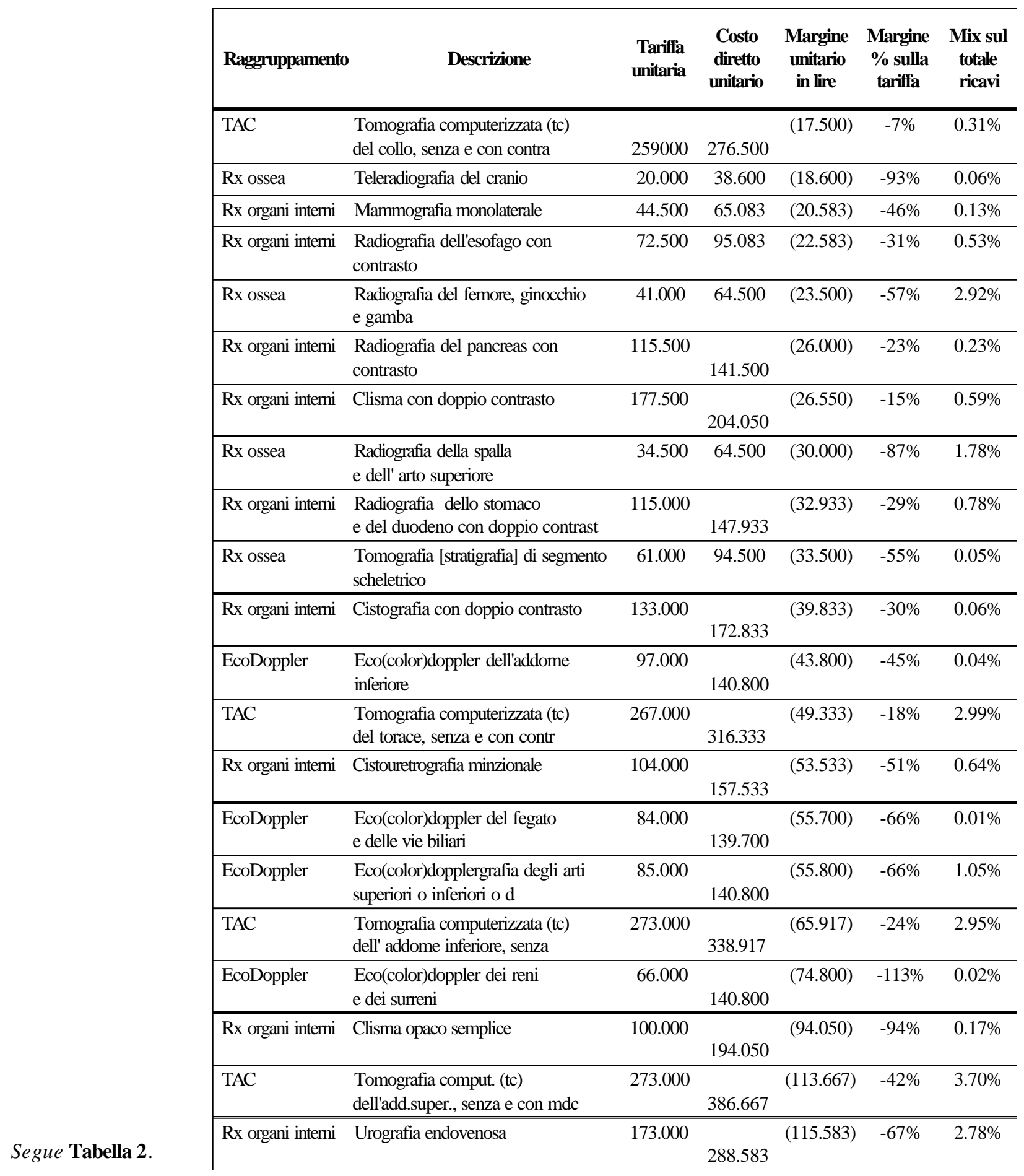

suggerirebbero di incrementare la produzione degli esami con margine unitario positivo e di ridurre la produzione degli esami con margine unitario negativo, in quanto:

- $\quad$ se il margine unitario è positivo, il beneficio economico complessivo sull' attività del reparto sarà tanto maggiore quanto più elevata è la quantità prodotta rispetto al mix complessivo di produzione;

- se il margine unitario è negativo, il danno economico complessivo sull' attività del reparto sarà tanto minore quanto più ridotta è la quantità prodotta rispetto al mix complessivo di produzione.
Ovviamente, trattandosi di azienda che produce un servizio pubblico, le possibilità di manovra in questo senso sono ridotte per una serie di considerazioni che limitano le leve di azione in merito a:

tipologie di esami che devono comunque essere garantite da una struttura pubblica, a maggior ragione se non contemplate nell'offerta delle strutture private (tipicamente gli esami meno redditizi);

quantità di esami offerta che non può scendere al di sotto di una determinata soglia, pena l'allungamento delle liste d'attesa soggette a monitoraggio regionale con conse- 


\begin{tabular}{|c|c|c|c|c|c|c|}
\hline \multicolumn{7}{|c|}{ CONTO ECONOMICO - valori unitari } \\
\hline raggrup. & $\begin{array}{c}\text { tariffa } \\
\text { media }\end{array}$ & $\begin{array}{c}\text { costo } \\
\text { diretto } \\
\text { unitario } \\
\text { medio }\end{array}$ & $\begin{array}{c}\text { margine } \\
\text { unitario } \\
\text { medio }\end{array}$ & $\begin{array}{l}\text { quant. } \\
\text { totali }\end{array}$ & margine $\%$ & $\begin{array}{l}\text { mix sul } \\
\text { totale } \\
\text { ricavi }\end{array}$ \\
\hline EcoDoppler & 84.888 & 133.503 & -48.615 & 456 & $-57 \%$ & $1.25 \%$ \\
\hline Ecografia & 79.920 & 58.635 & 21.285 & 6.323 & $27 \%$ & $16.27 \%$ \\
\hline RMN & 344.000 & 189.167 & 154.833 & 460 & $45 \%$ & $5.09 \%$ \\
\hline $\begin{array}{l}\text { Rx organi } \\
\text { interni }\end{array}$ & 44.134 & 49.009 & -4.874 & 18.948 & $-11 \%$ & $26.92 \%$ \\
\hline Rx ossea & 35.899 & 42.660 & -6.761 & 20.242 & $-19 \%$ & $23.40 \%$ \\
\hline TAC & 194.124 & 149.496 & 44.628 & 4.331 & $23 \%$ & $27.07 \%$ \\
\hline TOTALE & 61.189 & 58.279 & 2.910 & 50.760 & $5 \%$ & $100.00 \%$ \\
\hline \multicolumn{7}{|c|}{ CONTO ECONOMICO - valori totali } \\
\hline raggrup. & $\begin{array}{l}\text { ricavi } \\
\text { totali }\end{array}$ & $\begin{array}{c}\text { costo } \\
\text { diretto } \\
\text { totale }\end{array}$ & $\begin{array}{l}\text { margine } \\
\text { totale }\end{array}$ & $\begin{array}{l}\text { quant. } \\
\text { totali }\end{array}$ & $\begin{array}{l}\text { altri costi } \\
\text { di reparto }\end{array}$ & risultato \\
\hline EcoDoppler & 38.709 .000 & 60.877 .300 & $(22.168 .300)$ & 456 & & \\
\hline Ecografia & 505.336 .700 & 370.751 .933 & 134.584 .767 & 6.323 & & \\
\hline $\mathrm{RMN}$ & 158.240 .000 & 87.016 .667 & 71.223 .333 & 460 & & \\
\hline $\begin{array}{l}\text { Rx organi } \\
\text { interni }\end{array}$ & 836.258 .000 & 928.614 .050 & $(92.356 .050)$ & 18.948 & & \\
\hline Rx ossea & 726.668 .000 & 863.531 .800 & $(136.863 .800)$ & 20.242 & & \\
\hline TAC & 840.750 .500 & 647.467 .933 & 193.282 .567 & 4.331 & & \\
\hline TOTALE & 3.105 .962 .200 & 2.958 .259 .683 & 147.702 .517 & 50.760 & $(1.406 .230 .000)$ & $(1.258 .527 .483$ \\
\hline
\end{tabular}

Tabella 3

Conto economico di sintesi. guente insoddisfazione dell'utenza;

- esami per interni ricoverati presso l'ospedale che devono essere comunque eseguiti.

\section{Redditività per famiglia di prodotti}

Ciò premesso può risultare utile esaminare il contributo di ciascuna famiglia di prodotti al margine complessivo del periodo di riferimento ricordando che il margine complessivo di 147 milioni è comunque insufficiente alla copertura dei restanti costi non imputati al prodotto (pulizia, riscaldamento, costo del personale amministrativo, costo del personale tecnico per l'attività lavorativa indiretta, ecc.) che ammontano a 1.406 milioni.

I prodotti del raggruppamento Eco Doppler, contrariamente alle aspettative, presentano un margine totale negativo da addebitarsi principalmente agli alti tempi di esecuzione degli esami.

I prodotti del raggruppamento Ecografie presentano un margine totale positivo.

Il prodotto Risonanza Magnetica è quello che ha la tariffa più elevata (344.000 lire) tuttavia il margine è stato fortemente compresso nel periodo osservato dal ricorso ad un consulente esterno per l'esecuzione e la refertazione degli esami al costo di 300.000 lire/ora con l'effetto di comprimere il margine complessivo che pur rimane positivo.
I prodotti del raggruppamento $\mathrm{RX}$ organi interni e RX ossea presentano margini complessivi negativi dovuti al fatto che le prestazioni più erogate hanno margini ridottissimi (867 lire la radiografia del torace con quasi 13.000 prestazioni eseguite).

I prodotti della famiglia TAC presentano generalmente margini positivi.

Riportiamo inoltre nella Tabella 4 -Margine per prodotto- $\mathrm{i}$ valori analitici del margine per singolo prodotto: tali valori sono stati ordinati all'interno della famiglia di prodotto per quantità decrescenti di produzione in modo da evidenziare l'effetto moltiplicatorio delle quantità sul margine unitario di prodotto.

\section{Redditività per prodotto}

Approfondendo l'analisi e passando ad esaminare il contributo di ciascun prodotto al margine della famiglia di appartenenza si possono fare le seguenti considerazioni.

Quattro prodotti su cinque prodotti del raggruppamento Eco Doppler presentano margini unitari negativi e il prodotto che maggiormente contribuisce al margine complessivo (negativo) è l'Ecocolordopplergrafia degli arti inferiori o superiori con - 21,5 milioni di margine.

I prodotti del raggruppamento Ecografie presentano tutti un margine positivo ad eccezione della biopsia eco guidata e dell'esame 


\section{Figura 4}

Ricavi, costi diretti e margini complessivi per famiglia di prodotto.

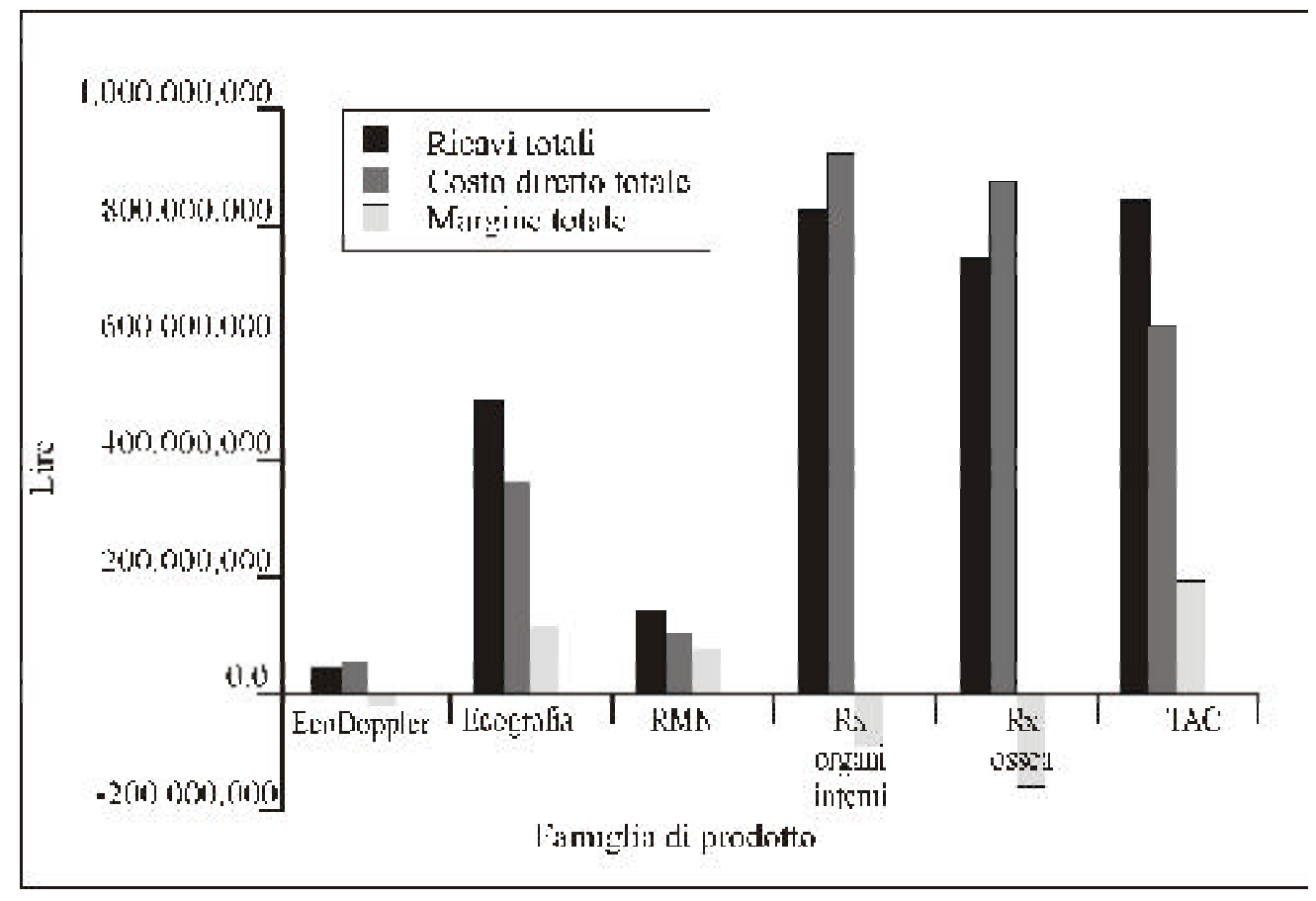

istocitopatologico della mammella che in relazione alle basse quantità prodotte penalizza il risultato complessivo in maniera ridotta $(-0,8$ milioni).

Nella categoria Risonanza Magnetica (eseguibile utilizzando la macchina esistente solo su piccole articolazioni) esiste un unico prodotto che presenta uno dei margini unitari più elevati $(45 \%)$ e un buon margine complessivo grazie alle 460 unità prodotte $(+71$ milioni di margine nell'anno oggetto di analisi: tale margine risulta ulteriormente incrementabile incrementando la quantità ed eliminando il ricorso al consulente esterno per eseguire l'esame in completa autonomia e a costi inferiori).

Il 67\% delle quantità prodotte (quasi 13.000 su 19.000) all'interno del raggruppamento RX organi interni è costituito da RX torace di routine che, prevedendo una tariffa di sole 30.000 lire, comporta un margine complessivo di soli 11 milioni. L'urografia endovenosa presenta il peggior margine unitario (-115.000) e il peggior margine complessivo (- 58 milioni), seguita da mammografia bilaterale (- 18 milioni), cistouretrografia minzionale (- 10 milioni) e dagli esami eseguiti con mezzo di contrasto. Complessivamente il margine del raggruppamento è negativo per 92 milioni.

La maggior parte dei prodotti di RX ossea presenta margini (sia unitari che complessivi) negativi, ma sono sufficienti la Radiografia del femore ginocchio e gamba (con - 52 milioni) e la Radiografia della spalla e dell' arto superiore (con - 48) per spiegare i - 132 milioni di margine totale. La tariffa media degli esami di questo raggruppamento è inferiore alle 36.000 lire.

I prodotti della famiglia TAC presentano generalmente margini positivi se si eccettuano le 3 TAC di addome superiore, inferiore e torace eseguite con mezzo di contrasto $(-48,-22$, e17 milioni). La tomografia del capo ha un margine positivo per quasi 100 milioni.

Prestazioni non adeguatamente remunerate

Rispetto all'attività svolta ordinariamente dal reparto, occorre ancora effettuare alcune precisazioni in merito a prestazioni che possono comportare impieghi unitari di risorse maggiori rispetto a quelli standard in termini di tempo necessario nei seguenti casi:

esami per interni effettuati nel reparto di degenza con l'ausilio di apparecchiature portatili riguardanti per i quali è sconsigliato il trasporto;

esami effettuati per il Pronto Soccorso su pazienti poli traumatizzati per i quali è necessaria la massima cautela.

Èinoltre da prendere in considerazione tutta l'attività di screening in relazione alle campagne per la prevenzione dei tumori femminili che prevede un elevato numero annuo di prestazioni di primo livello e un limitato ma ben più impegnativo numero di prestazioni di secondo livello, che vengono remunerate a parte. Per alcune di queste prestazioni è prevista la doppia lettura con un consumo di risorse superiore rispetto agli standard. vista, gli esami di mammografia presentano un margine negativo per cui comportano comunque una perdita unitaria di circa 11.000 lire.

Per quanto riguarda la radiologia ossea, sono inoltre frequenti le richieste di esami da eseguirsi mediante l'osservazione di particola-
Oltretutto, seppur remunerati alla tariffa pre- 


\begin{tabular}{|c|c|c|c|c|c|c|c|c|}
\hline Raggrup. & Cod & Descrizione & $\begin{array}{r}\text { Tariffa } \\
\text { unitaria }\end{array}$ & $\begin{array}{r}\text { Costo } \\
\text { diretto } \\
\text { unitario } \\
\end{array}$ & $\begin{array}{c}\text { Margine } \\
\text { unitario } \\
\text { in lire }\end{array}$ & $\begin{array}{l}\text { Margine } \\
\text { \% sulla } \\
\text { tariffa }\end{array}$ & $\begin{array}{l}\text { Totale } \\
\text { quantità }\end{array}$ & $\begin{array}{c}\text { Margine } \\
\text { totale }\end{array}$ \\
\hline \multirow[t]{5}{*}{ EcoDoppler } & 105 & $\begin{array}{l}\text { Eco(color)dopplergrafia } \\
\text { degli arti superiori o } \\
\text { inferiori o d }\end{array}$ & 85.000 & 140.800 & (55.800) & $-66 \%$ & 385 & (21.483.000) \\
\hline & 102 & $\begin{array}{l}\text { Eco(color)doppler dei } \\
\text { tronchi sovraaortici }\end{array}$ & 85.000 & 65.300 & 19.700 & $23 \%$ & 44 & 866.800 \\
\hline & 104 & $\begin{array}{l}\text { Eco(color)doppler } \\
\text { dell'addome inferiore }\end{array}$ & 97.000 & 140.800 & (43.800) & $-45 \%$ & 12 & $(525.600)$ \\
\hline & 101 & $\begin{array}{l}\text { Eco(color)doppler dei } \\
\text { reni e dei surreni }\end{array}$ & 66.000 & 140.800 & (74.800) & $-113 \%$ & 10 & $(748.000)$ \\
\hline & 103 & $\begin{array}{l}\text { Eco(color)doppler del } \\
\text { fegato e delle vie biliari }\end{array}$ & 84.000 & 139.700 & $(55.700)$ & $-66 \%$ & 5 & (278.500) \\
\hline \multicolumn{2}{|c|}{ Totale EcoDoppler } & & 84.888 & & & $-57 \%$ & 456 & $(22.168 .300)$ \\
\hline \multirow[t]{17}{*}{ Ecografia } & 212 & $\begin{array}{l}\text { Ecografia dell' addome } \\
\text { superiore }\end{array}$ & 85.000 & 59.133 & 25.867 & $30 \%$ & 1.625 & 42.033 .333 \\
\hline & 208 & $\begin{array}{l}\text { Ecografia addome } \\
\text { completo }\end{array}$ & 117.000 & 69.200 & 47.800 & $41 \%$ & 1.253 & 59.893 .400 \\
\hline & 211 & $\begin{array}{l}\text { Ecografia dell' addome } \\
\text { inferiore }\end{array}$ & 62.000 & 59.133 & 2.867 & $5 \%$ & 706 & 2.023 .867 \\
\hline & 214 & $\begin{array}{l}\text { Ecografia della } \\
\text { mammella }\end{array}$ & 69.500 & 48.950 & 20.550 & $30 \%$ & 665 & 13.665 .750 \\
\hline & 206 & $\begin{array}{l}\text { Diagnostica ecografica } \\
\text { del capo e del collo }\end{array}$ & 55.000 & 48.950 & 6.050 & $11 \%$ & 612 & 3.702 .600 \\
\hline & 218 & $\begin{array}{l}\text { Ecografia } \\
\text { osteoarticolare }\end{array}$ & 63.000 & 59.133 & 3.867 & $6 \%$ & 569 & 2.200 .133 \\
\hline & 219 & Ecografia transrettale & 84.000 & 59.133 & 24.867 & $30 \%$ & 303 & 7.534 .600 \\
\hline & 213 & $\begin{array}{l}\text { Ecografia della cute e } \\
\text { del tessuto } \\
\text { sottocutaneo }\end{array}$ & 55.000 & 48.950 & 6.050 & $11 \%$ & 203 & 1.228 .150 \\
\hline & 217 & $\begin{array}{l}\text { Ecografia } \\
\text { muscolotendinea }\end{array}$ & 55.000 & 48.950 & 6.050 & $11 \%$ & 174 & 1.052 .700 \\
\hline & 209 & Ecografia dei testicoli & 60.000 & 48.950 & 11.050 & $18 \%$ & 92 & 1.016 .600 \\
\hline & 204 & $\begin{array}{l}\text { Biopsia eco-guidata } \\
\text { della mammella }\end{array}$ & 72.000 & 84.783 & (12.783) & $-18 \%$ & 52 & $(664.733)$ \\
\hline & 215 & $\begin{array}{l}\text { Ecografia di grossi vasi } \\
\text { addominali }\end{array}$ & 62.000 & 59.133 & 2.867 & $5 \%$ & 26 & 74.533 \\
\hline & 203 & $\begin{array}{l}\text { Biopsia [percutanea] } \\
\text { [agobiopsia] della } \\
\text { tiroide }\end{array}$ & 119.000 & 69.200 & 49.800 & $42 \%$ & 15 & 747.000 \\
\hline & 220 & $\begin{array}{l}\text { Es. istocitopatologico } \\
\text { mammella: biopsia } \\
\text { stereotassica }\end{array}$ & 90.000 & 102.367 & (12.367) & $-14 \%$ & 10 & (123.667) \\
\hline & 205 & $\begin{array}{l}\text { Biopsia eco-guidata } \\
\text { dei tessuti molli }\end{array}$ & 91.800 & 69.200 & 22.600 & $25 \%$ & 9 & 203.400 \\
\hline & 210 & Ecografia del pene & 60.000 & 55.883 & 4.117 & $7 \%$ & 6 & 24.700 \\
\hline & 216 & Ecografia ginecologica & 60.000 & 69.200 & $(9.200)$ & $-15 \%$ & 3 & $(27.600)$ \\
\hline \multicolumn{2}{|c|}{ Totale Ecografia } & & 79.920 & & & $27 \%$ & 6.323 & 134.584 .767 \\
\hline RMN & 301 & $\begin{array}{l}\text { Risonanza magnetica } \\
\text { nucleare }(\mathrm{rm}) \\
\text { muscoloscheletrica }\end{array}$ & 344.000 & 189.167 & 154.833 & $45 \%$ & 460 & 71.223 .333 \\
\hline Totale RMN & & & 344.000 & & & $45 \%$ & 460 & 71.223 .333 \\
\hline \multirow[t]{6}{*}{ Rx org.interni } & 422 & $\begin{array}{l}\text { Radiografia del torace } \\
\text { di routine, nas }\end{array}$ & 30.000 & 29.133 & 867 & $3 \%$ & 12.748 & 11.048 .267 \\
\hline & 413 & $\begin{array}{l}\text { Mammografia } \\
\text { bilaterale }\end{array}$ & 67.500 & 77.667 & (10.167) & $-15 \%$ & 1.731 & (17.598.500) \\
\hline & 423 & Radiografia addome & 37.500 & 44.733 & $(7.233)$ & $-19 \%$ & 1.039 & (7.515.433) \\
\hline & 424 & $\begin{array}{l}\text { Radiografia } \\
\text { apparato urinario }\end{array}$ & 37.500 & 35.733 & 1.767 & $5 \%$ & 810 & 1.431 .000 \\
\hline & 432 & Urografia endovenosa & 173.000 & 288.583 & (115.583) & $-67 \%$ & 500 & (57.791.667) \\
\hline & 427 & $\begin{array}{l}\text { Telecuore con esofago } \\
\text { baritato }\end{array}$ & 61.000 & 45.400 & 15.600 & $26 \%$ & 489 & 7.628 .400 \\
\hline
\end{tabular}

Tabella 4

Margine per prodotto. 
La valutazione dei costi: uno studio applicato all'unità operativa di diagnostica per immagini

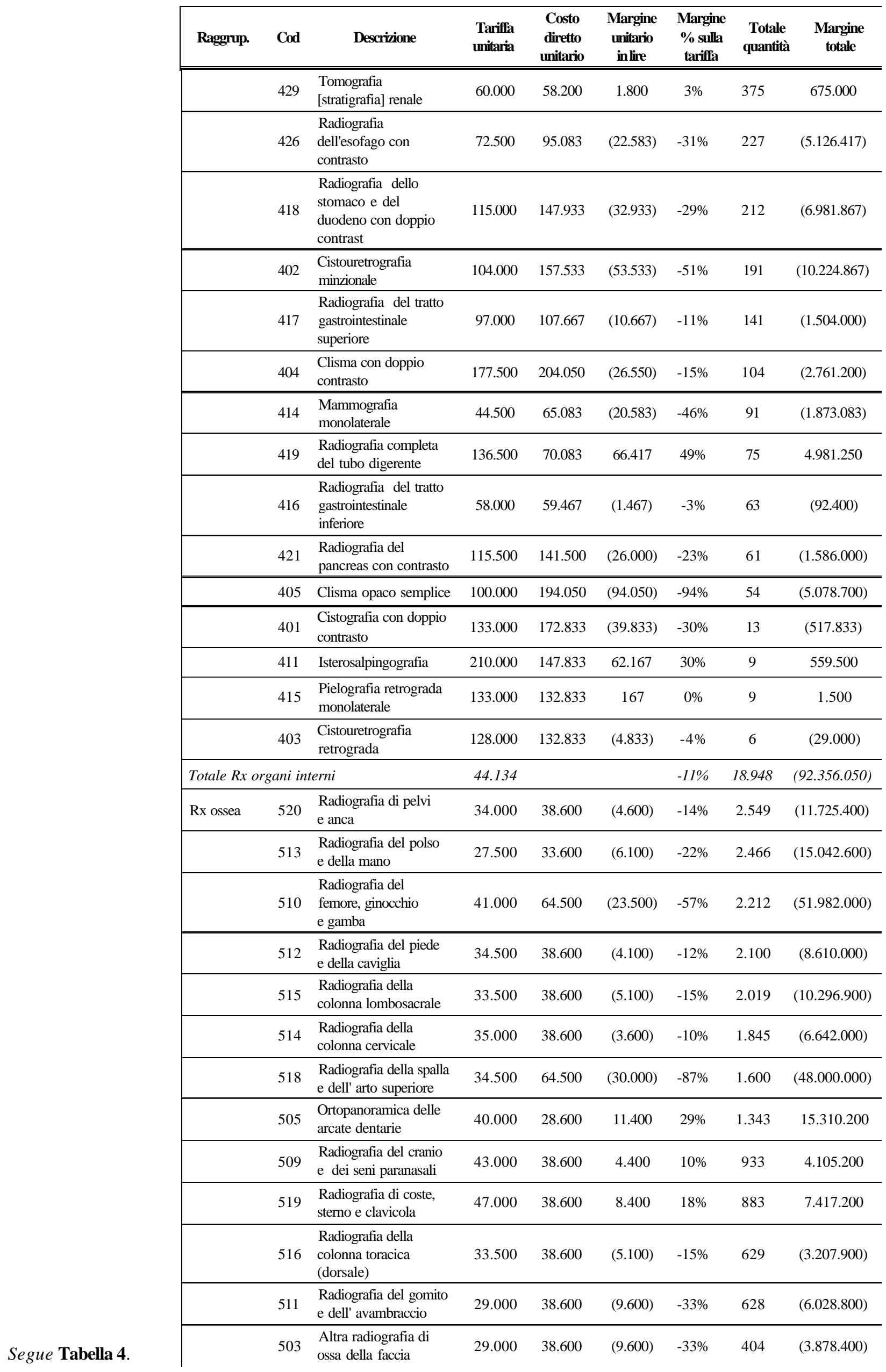




\begin{tabular}{|c|c|c|c|c|c|c|c|c|}
\hline Raggrup. & Cod & Descrizione & $\begin{array}{l}\text { Tariffa } \\
\text { unitaria }\end{array}$ & $\begin{array}{c}\text { Costo } \\
\text { diretto } \\
\text { unitario }\end{array}$ & $\begin{array}{c}\text { Margine } \\
\text { unitario } \\
\text { in line }\end{array}$ & $\begin{array}{c}\text { Margine } \\
\% \text { sulla } \\
\text { tariffa }\end{array}$ & $\begin{array}{l}\text { Totale } \\
\text { quantità }\end{array}$ & $\begin{array}{c}\text { Margine } \\
\text { totale }\end{array}$ \\
\hline & 508 & $\begin{array}{l}\text { Radiografia completa } \\
\text { della colonna }\end{array}$ & 67.000 & 56.667 & 10.333 & $15 \%$ & 363 & 3.751 .000 \\
\hline & 522 & $\begin{array}{l}\text { Teleradiografia del } \\
\text { cranio }\end{array}$ & 20.000 & 38.600 & $(18.600)$ & $-93 \%$ & 87 & $(1.618 .200)$ \\
\hline & 506 & $\begin{array}{l}\text { Radiografia assiale } \\
\text { della rotula }\end{array}$ & 51.000 & 38.600 & 12.400 & $24 \%$ & 83 & 1.029 .200 \\
\hline & 507 & $\begin{array}{l}\text { Radiografia completa } \\
\text { degli arti inferiori e del } \\
\text { bacino sotto }\end{array}$ & 58.500 & 64.500 & $(6.000)$ & $-10 \%$ & 48 & $(288.000)$ \\
\hline & 524 & $\begin{array}{l}\text { Tomografia } \\
\text { [stratigrafia] di } \\
\text { segmento scheletrico }\end{array}$ & 61.000 & 94.500 & $(33.500)$ & $-55 \%$ & 26 & $(871.000)$ \\
\hline & 521 & Studio dell' eta' ossea & 23.500 & 38.600 & $(15.100)$ & $-64 \%$ & 17 & $(256.700)$ \\
\hline & 517 & $\begin{array}{l}\text { Radiografia della sella } \\
\text { turcica }\end{array}$ & 29.500 & 33.600 & $(4.100)$ & $-14 \%$ & 7 & $(28.700)$ \\
\hline \multicolumn{2}{|c|}{ Totale Rx ossea } & & 35.899 & & & $-19 \%$ & 20.242 & (136.863.800) \\
\hline \multirow[t]{14}{*}{ TAC } & 610 & $\begin{array}{l}\text { Tomografia } \\
\text { computerizzata (tc) del } \\
\text { capo }\end{array}$ & 161.000 & 47.400 & 113.600 & $71 \%$ & 872 & 99.059 .200 \\
\hline & 604 & $\begin{array}{l}\text { Tomografia } \\
\text { computerizzata (tc) } \\
\text { del rahide e dello } \\
\text { speco ve }\end{array}$ & 167.000 & 102.333 & 64.667 & $39 \%$ & 533 & 34.467 .333 \\
\hline & 603 & $\begin{array}{l}\text { Tomografia comput. } \\
\text { (tc) dell'add.super., } \\
\text { senza e con mdc }\end{array}$ & 273.000 & 386.667 & $(113.667)$ & $-42 \%$ & 421 & (47.853.667) \\
\hline & 601 & $\begin{array}{l}\text { Densitometria ossea } \\
\text { con tc }\end{array}$ & 149.000 & 41.083 & 107.917 & $72 \%$ & 396 & 42.735 .000 \\
\hline & 618 & $\begin{array}{l}\text { Tomografia } \\
\text { computerizzata (tc) } \\
\text { del torace, senza } \\
\text { e con contr } \\
\end{array}$ & 267.000 & 316.333 & (49.333) & $-18 \%$ & 348 & $(17.168 .000)$ \\
\hline & 622 & $\begin{array}{l}\text { Tomografia } \\
\text { computerizzata (tc) } \\
\text { dell' addome inferiore, } \\
\text { senza }\end{array}$ & 273.000 & 338.917 & (65.917) & $-24 \%$ & 336 & $(22.148 .000)$ \\
\hline & 616 & $\begin{array}{l}\text { Tomografia } \\
\text { computerizzata (tc) } \\
\text { del rachide } \\
\text { e dello speco ver }\end{array}$ & 57.000 & 37.167 & 19.833 & $35 \%$ & 333 & 6.604 .500 \\
\hline & 611 & $\begin{array}{l}\text { Tomografia } \\
\text { computerizzata (tc) } \\
\text { del capo, senza } \\
\text { e con contras } \\
\end{array}$ & 259.000 & 184.533 & 74.467 & $29 \%$ & 239 & 17.797 .533 \\
\hline & 617 & $\begin{array}{l}\text { Tomografia } \\
\text { computerizzata (tc) } \\
\text { del torace }\end{array}$ & 167.000 & 120.000 & 47.000 & $28 \%$ & 188 & 8.836 .000 \\
\hline & 624 & $\begin{array}{l}\text { Tomografia } \\
\text { computerizzata (tc) } \\
\text { dell' arto inferiore }\end{array}$ & 167.500 & 76.667 & 90.833 & $54 \%$ & 147 & 13.352 .500 \\
\hline & 614 & $\begin{array}{l}\text { Tomografia } \\
\text { computerizzata (tc) } \\
\text { del massiccio facciale }\end{array}$ & 173.000 & 84.500 & 88.500 & $51 \%$ & 126 & 11.151 .000 \\
\hline & 620 & $\begin{array}{l}\text { Tomografia } \\
\text { computerizzata (tc) } \\
\text { dell' addome } \\
\text { completo, senza }\end{array}$ & 340.000 & 80.583 & 259.417 & $76 \%$ & 108 & 28.017 .000 \\
\hline & 623 & $\begin{array}{l}\text { Tomografia } \\
\text { computerizzata (tc) } \\
\text { dell' addome superiore }\end{array}$ & 171.000 & 73.333 & 97.667 & $57 \%$ & 75 & 7.325 .000 \\
\hline & 625 & $\begin{array}{l}\text { Tomografia } \\
\text { computerizzata (tc) } \\
\text { dell' orecchio }\end{array}$ & 161.000 & 104.917 & 56.083 & $35 \%$ & 53 & 2.972 .417 \\
\hline
\end{tabular}

Segue Tabella 4. 


\begin{tabular}{|c|c|c|c|c|c|c|c|c|c|}
\hline & Raggrup. & Cod & Descrizione & $\begin{array}{r}\text { Tariffa } \\
\text { unitaria }\end{array}$ & $\begin{array}{c}\text { Costo } \\
\text { diretto } \\
\text { unitario }\end{array}$ & $\begin{array}{c}\text { Margine } \\
\text { unitario } \\
\text { inlire } \\
\end{array}$ & $\begin{array}{c}\text { Margine } \\
\% \text { sulla } \\
\text { tariffa }\end{array}$ & $\begin{array}{c}\text { Totale } \\
\text { quantità }\end{array}$ & $\begin{array}{c}\text { Margine } \\
\text { totale }\end{array}$ \\
\hline & & 613 & $\begin{array}{l}\text { Tomografia } \\
\text { computerizzata (tc) } \\
\text { del collo, senza e con } \\
\text { contra }\end{array}$ & 259.000 & 276.500 & $(17.500)$ & $-7 \%$ & 37 & $(647.500)$ \\
\hline & & 612 & $\begin{array}{l}\text { Tomografia } \\
\text { computerizzata (tc) } \\
\text { del collo }\end{array}$ & 161.000 & 66.667 & 94.333 & $59 \%$ & 31 & 2.924 .333 \\
\hline & & 615 & $\begin{array}{l}\text { Tomografia } \\
\text { computerizzata (tc) } \\
\text { del massiccio facciale, } \\
\text { senza }\end{array}$ & 275.000 & 285.500 & $(10.500)$ & $-4 \%$ & 26 & $(273.000)$ \\
\hline & & 608 & $\begin{array}{l}\text { Tomografia } \\
\text { computerizzata (tc) } \\
\text { dei reni, senza } \\
\text { e con contras }\end{array}$ & 289.000 & 183.917 & 105.083 & $36 \%$ & 16 & 1.681 .333 \\
\hline & & 626 & $\begin{array}{l}\text { Tomografia } \\
\text { computerizzata (tc) } \\
\text { dell' orecchio, senza } \\
\text { e con c }\end{array}$ & 259.000 & 202.583 & 56.417 & $22 \%$ & 14 & 789.833 \\
\hline & & 619 & $\begin{array}{l}\text { Tomografia } \\
\text { computerizzata (tc) } \\
\text { dell' addome completo }\end{array}$ & 223.000 & 93.167 & 129.833 & $58 \%$ & 10 & 1.298 .333 \\
\hline & & 605 & $\begin{array}{l}\text { Tomografia } \\
\text { computerizzata (tc) } \\
\text { dell' arto superiore }\end{array}$ & 176.000 & 60.750 & 115.250 & $65 \%$ & 9 & 1.037 .250 \\
\hline & & 621 & $\begin{array}{l}\text { Tomografia } \\
\text { computerizzata (tc) } \\
\text { dell' addome inferiore }\end{array}$ & 171.000 & 73.333 & 97.667 & $57 \%$ & 7 & 683.667 \\
\hline & & 609 & $\begin{array}{l}\text { Tomografia } \\
\text { computerizzata (tc) } \\
\text { del bacino }\end{array}$ & 167.500 & 60.750 & 106.750 & $64 \%$ & 6 & 640.500 \\
\hline & Totale TAC & & & 194.124 & & & $23 \%$ & 4.331 & 193.282.567 \\
\hline Segue Tabella 4. & Totale comp & ssivo & & 61.189 & & & $5 \%$ & 50.760 & 147.702 .517 \\
\hline
\end{tabular}

ri procedure (es. protocollo del ginocchio) che a fronte di una remunerazione media di 36.000 lire comportano l'impiego tempi notevoli.

Un'ultima attività non adeguatamente remunerata è quella relativa alle "copie" di pellicole effettuate per esterni e per interni.

9) Attribuzione ai prodotti degli ammortamenti calcolati in base al costo di sostituzione e al periodo di vita utile delle macchine

Per consentire l'espressione di valutazioni mediante il calcolo di un margine al netto degli ammortamenti, sono state censite le macchine strumentali a disposizione del reparto di Radiologia per le quali è stato rilevato il costo storico e l'anno di acquisizione.

Al fine di effettuare simulazioni ed analisi prospettiche si è ritenuto che l'utilizzo dei costi delle macchine esistenti per il calcolo degli ammortamenti avrebbe potuto portare a valutazioni fuorvianti per i seguenti motivi:

- il costo di riferimento è quello storico che potrebbe non corrispondere al costo di sostituzione, soprattutto per le macchine acquisite molti anni addietro;

- gran parte delle macchine risulta completamente ammortizzata (dal punto di vista con- tabile) e una certa parte è al termine del periodo di vita utile per cui la redditività di un prodotto così calcolata potrebbe essere solo apparente e venir meno in caso di acquisto di una macchina nuova.

Il valore degli ammortamenti è perciò stato calcolato secondo le seguenti ipotesi:

costo di sostituzione, relativo all'acquisto di macchine al livello tecnologico corrente; periodo di ammortamento definito in base alla vita utile delle macchine (tenendo conto sia della senescenza che dell'obsolescenza) e imputato in base al numero effettivo di esami.

In particolare l'adozione dei criteri su esposti ha comportato per i prodotti appartenenti ad alcune famiglie una aggregazione ad un livello intermedio in coerenza col tipo di macchina utilizzata. Èil caso della famiglia che contiene le prestazioni di RX organi interni, che è stata distinta nelle sottoaggregazioni RX torace, Mammografie e altri RX organi interni, e della famiglia RX ossea dalla quale sono state isolate le prestazioni di RX arcate dentarie che utilizzano l'ortopantomografo.

Nella Tabella 5 -Ipotesi per ammortamentisono stati riportati i dati generali utilizzati per 


\begin{tabular}{|llccccc}
\hline \multicolumn{1}{|c}{ Macchina } & Utilizzo & $\begin{array}{c}\text { Costo } \\
\text { unitario } \\
\text { Sostituz. } \\
\text { (mil.) }\end{array}$ & $\begin{array}{c}\text { Quantità } \\
\text { necessaria }\end{array}$ & $\begin{array}{c}\text { Costo } \\
\text { quantità } \\
\text { (mil.) }\end{array}$ & $\begin{array}{c}\text { Durata } \\
\text { utile } \\
\text { (anni) }\end{array}$ & $\begin{array}{c}\text { Costo } \\
\text { totale } \\
\text { annuo } \\
\text { (mil.) }\end{array}$ \\
\hline \hline $\begin{array}{l}\text { ecografo } \\
\text { Ecografia }\end{array}$ & $\begin{array}{l}\text { Eco } \\
\text { Doppler }\end{array}$ & 300 & 2 & 600 & 7 & 86 \\
\hline \hline $\begin{array}{l}\text { risonanza } \\
\text { magnetica }\end{array}$ & RMN & 450 & 1 & 450 & 5 & 90 \\
\hline $\begin{array}{l}\text { chest } \\
\text { (rx torace) }\end{array}$ & $\begin{array}{l}\text { Rx organi } \\
\text { interni }\end{array}$ & 120 & 1 & 120 & 10 & 12 \\
\hline mammografo & $\begin{array}{l}\text { Rx organi } \\
\text { interni }\end{array}$ & 130 & 1 & 130 & 8 & 16 \\
\hline telecomandato & $\begin{array}{l}\text { Rx organi } \\
\text { interni }\end{array}$ & 400 & 2 & 800 & 10 & 80 \\
\hline $\begin{array}{l}\text { ortopanto- } \\
\text { mografo }\end{array}$ & Rx ossea & 40 & 1 & 40 & 10 & 4 \\
\hline trocoscopio & Rx ossea & 100 & 1 & 100 & 10 & 10 \\
\hline tomografo & TAC & 900 & 1 & 900 & 8 & 113 \\
\hline
\end{tabular}

Tabella 5

Ipotesi per ammortamenti. ogni macchina radiologica.

Imputando il costo dell' ammortamento annuo in misura proporzionale agli esami eseguiti da ciascuna macchina, è stato possibile determinare un margine complessivo per famiglia di prodotto al netto degli ammortamenti specifici del tipo di macchina utilizzata in modo da consentire valutazioni prospettiche, come riportato nella Tabella 6 .

Tali dati sono di fondamentale importanza al fine di costruire delle simulazioni ipotizzando la variazione del numero di esami per ogni famiglia e dei relativi costi e ricavi mantenendo costante il valore complessivo degli ammortamenti (entro la massima capacità produttiva della macchina).

Inoltre è stato possibile determinare il punto di pareggio per ciascuna famiglia di prodotti (con margine positivo) tale da coprire il costo dell'ammortamento alle condizioni standard, che corrisponde:

- per le ecografie (escluso eco doppler) a 3.756 esami/anno, (eseguiti nell'anno in esame: 6.323);

- per le risonanze magnetiche a 581 esami/ anno (eseguiti nell' anno considerato: 460);

- per i soli RX torace (all'interno della famiglia rx organi interni) a 13.846 esami/anno (eseguiti nell'anno in esame: 12.748);

- per i soli RX arcate dentarie (all'interno della famiglia rx ossea) a 351 esami/anno, (eseguiti nell' anno osservato 1.343 );

- per le TAC a 2.521 esami anno, (eseguiti nell' anno in esame: 4.331);

- mentre per eco doppler altri RX organi interni e ossea (esclusi torace e arcate dentarie) non esiste punto di pareggio in quanto, essendo il margine unitario negativo, ogni quantità prodotta in più, anziché coprire il costo dell'ammortamento, non fa che aumentare il disavanzo.

10) Ipotesi di miglioramento e simulazione delle possibili conseguenze economiche

Rispetto a tale quadro di analisi è stata effettuata un' analisi allo scopo di verificare l'impatto economico delle seguenti linee d'azione: - verifica della compatibilità di possibili incrementi di produzione con le saturazioni delle macchine, degli spazi fisici, e del personale addetto;

miglioramento dell'efficienza delle prestazioni a margine negativo;

- riorientamento del mix di produzione, in particolare per quanto attiene alle prestazioni per esterni, verso i prodotti più redditizi;

effettuazione in autonomia delle prestazioni di risonanza magnetica.

Emergono le seguenti considerazioni:

\section{Saturazione dei fattori produttivi}

Tra tutti i fattori produttivi impiegati nell'esecuzione degli esami (macchina, spazio fisico, pellicole e materiali di consumo, tempo degli operatori) il vero fattore che limita incrementi di produzione risulta essere il tempo dei medici, essendo questo il fattore che maggiormente incide sul costo unitario delle prestazioni.

Le TAC senza contrasto e la Risonanza magnetica possono essere eseguite anche dal solo Tecnico, ma risulta arduo reperire personale tecnico con esperienza in merito.

Pertanto qualsiasi simulazione deve tendere a non modificare eccessivamente l'impegno complessivo dei medici al fine di non introdurre nuovi elementi di costo qualora il personale medico risultasse non più sufficiente. 
Eco doppler ed ecografie

È consigliabile la riduzione delle prestazioni di Eco Doppler in quanto a margine negativo, mentre sono da incrementare tutte le altre ecografie in quanto a margine positivo.

Le ecografie inoltre presentano uno strano fenomeno per cui più ne vengono effettuate e più le liste di attesa si allungano, e anche il numero di prestazioni effettuate da studi privati non decresce con le ovvie conseguenze sul bilancio dell'ASL.

\section{Risonanza Magnetica}

L'effettuazione in autonomia delle prestazioni di risonanza magnetica porterebbe il margine unitario da 155.000 a 210.000 lire: il tecnico può eseguire la prestazione e il medico refertarla. Inoltre un sostanzioso incremento del numero di tali prestazioni avrebbe effetti sicuramente positivi. Il punto di pareggio per la copertura dell' ammortamento è rappresentato da 581 prestazioni e nell' anno esaminato ne sono state eseguite 460 .

Con 920 prestazioni il margine al netto dell'ammortamento sarebbe di 200 milioni.

Radiologia tradizionale (organi interni e ossea)

Tali prestazioni sono, in linea di massima, da contenere il più possibile.

Devono essere incrementati almeno sino a $14.000 \mathrm{i} \mathrm{RX}$ torace in modo da raggiungere il punto di pareggio.

Potrebbe anche risultare conveniente un incremento delle radiografie delle arcate dentarie ma, allo stato attuale, la domanda risulta più che soddisfatta.

Per le prestazioni di Urografia endovenosa (RX organi interni) esiste un eccesso di prescrizioni: nell'ipotesi di dimezzare il numero di prestazioni il margine di prodotto passerebbe da -58 a -29 milioni.

\section{$T A C$}

Queste prestazioni sono da incrementare in quanto generalmente a margine positivo. È da valutare l'ipotesi di acquisire una nuova TAC spirale che permetterebbe la riduzione dei tem- pi di esecuzione e del consumo di materiale di contrasto. Un investimento di 900 milioni nell'ipotesi di effettuare 5.000 esami anno per 8 anni graverebbe unitariamente sul prodotto per 22.500 lire.

La prestazione di densitometria ossea ha un margine molto elevato ( $72 \%$ ) e le 396 prestazioni eseguite hanno portato un margine positivo di 43 milioni. Tale prestazione è al momento stata limitata ad una determinata fascia di età ma sono molte le richieste da parte di persone di età superiore. Tale fascia potrebbe essere ampliata ed ipotizzando un incremento delle prestazioni del $30 \%$ il margine complessivo passerebbe da 43 a 56 milioni.

\section{Simulazione}

È stato quindi possibile effettuare numerose simulazioni al fine di valutare l'impatto economico di diverse alternative d'azione. A titolo di esempio si riporta la simulazione relativa alle seguenti ipotesi:

- Ore medici totali non superiori a quelle dell'anno precedente;

- Eco Doppler: max 228 prestazioni (-50\%);

- Ecografia: min 8.000 prestazioni (+26\%);

- Risonanza Magnetica: medico al posto del consulente, min 920 prestazioni $(+100 \%)$;

RX Organi interni: complessivamente max 18.100 prestazioni (-14\%), RX torace $\min 15$ 000 prestazioni $(+18 \%)$, Urografie $\max 250$ prestazioni $(-50 \%)$;

- RX Ossea: max 15.182 prestazioni (-25\%); TAC: complessivamente min 5.237 prestazioni (+20\%), Densitometria ossea min 515 prestazioni $(+30 \%)$;

- Riduzione sul costo del personale indiretto (- 50 milioni).

Il risultato della simulazione è riportato nella Tabella 7 -Simulazione economica-. Come si può osservare il margine complessivo, pur restando negativo, migliora di circa 350 milioni, ma di fronte al vincolo di non incrementare l'impegno in termini di ore, e il conseguente costo, del personale medico questo risultato appare come un discreto miglioramento in quanto il margine negativo passa dal $41 \%$ dei ricavi al $28 \%$. 